\title{
Le XXIe convoi : études biographiques (Première partie)
}

\author{
Insa Meinen et Ahlrich Meyer
}

\section{(2) OpenEdition \\ 1 Journals}

\section{Édition électronique}

URL : http://journals.openedition.org/cmc/796

DOI : $10.4000 / \mathrm{cmc} .796$

ISSN : 2684-3080

\section{Éditeur}

Fondation de la Mémoire Contemporaine

\section{Édition imprimée}

Date de publication : 1 janvier 2006

Pagination : 57-109

ISSN : 1377-1256

\section{Référence électronique}

Insa Meinen et Ahlrich Meyer, «Le XXle convoi : études biographiques (Première partie) », Les Cahiers de la Mémoire Contemporaine [En ligne], 7 | 2006, mis en ligne le 01 octobre 2020, consulté le 30 octobre 2020. URL : http://journals.openedition.org/cmc/796 ; DOI : https://doi.org/10.4000/cmc.796 


\section{Le XXIe convoi : études biographiques (Première partie)}

\section{Insa Meinen - Ahlrich Meyer}

Pourquoi une étude sur un convoi de déportation vers A uschwitz ?* À quoi bon une recherche portant sur l'un des 27 convois par lesquels les Allemands transférèrent les J uifs arrêtés en Belgique au cours de la Seconde Guerre mondiale sur les lieux où ils devaient mourir? L'histoire du judéocide en Belgique est écrite depuis longtemps surtout grâce aux efforts de Maxime Steinberg, dont personne ne pourra dépasser la recherche. Néanmoins, nous en savons toujours bien trop peu touchant les hommes, les femmes et les enfants de B elgique tués à Auschwitz et les circonstances de leur déportation. La constatation vaut aussi à propos de questions centrales. Comment les J uifs tombèrent-ils aux mains de la Sicherheitspolizei ? Comment avaient-ils

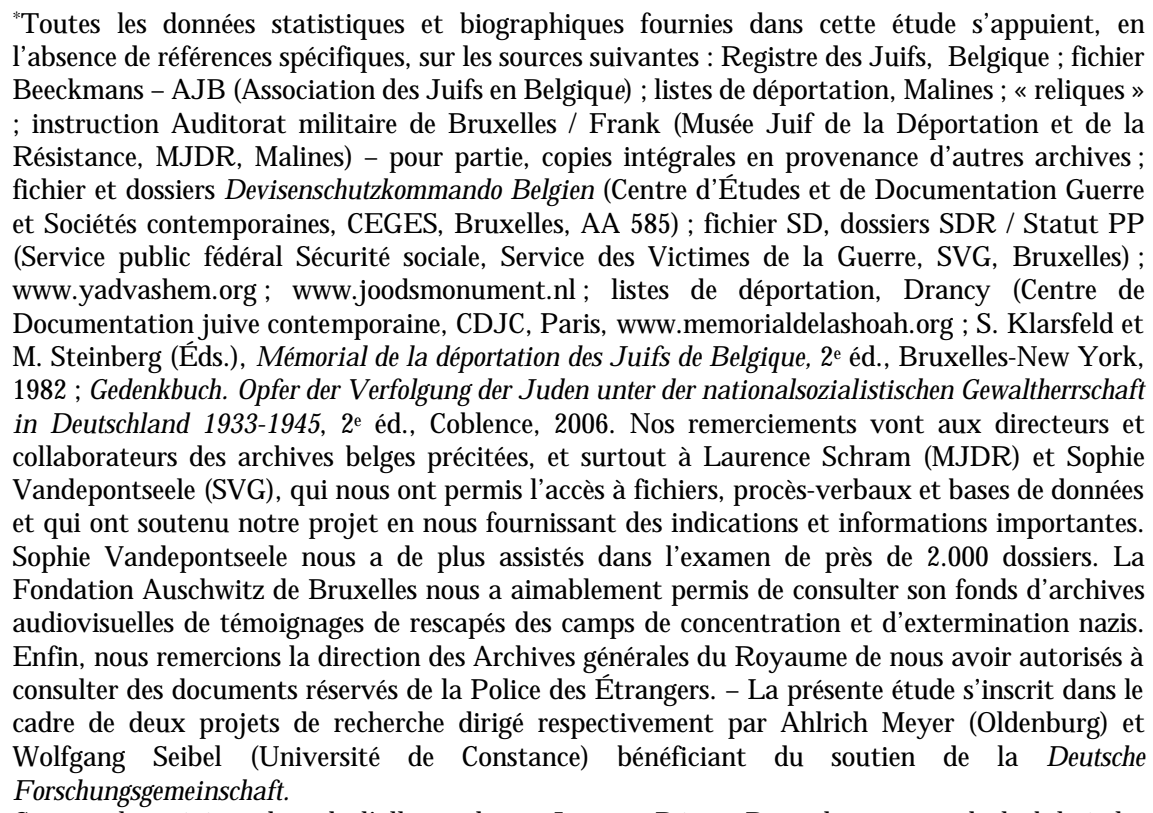
Cet article a été traduit de l'allemand par J acques Déom. Pour des raisons de lisibilité, les citations ont également fait l'objet d'une traduction. 
tenté de se protéger de la déportation ? Quelle expérience de l'exil les déportés avaient-ils déjà derrière eux ? Quelle importance les stratégies de survie des victimes eurent-elles sur la technique et l'ampleur des arrestations ? C'est là que se situe le point de départ de notre enquête.

Commençons par la méthode employée par les Allemands. Les grandes opérations d'arrestation sont il est vrai bien étudiées. Mais la majeure partie des J uifs déportés de B elgique à A uschwitz entre 1942 et 1944 - contrairement à ce qui se passa en F rance ou aux Pays-B as fut arrêtée non lors de grandes rafles ou autres arrestations de masse, mais individuellement ou en petits groupes ${ }^{1}$. I I reste donc nécessaire de tirer au clair qui a effectué ces arrestations, quels services allemands contribuèrent à la déportation des J uifs de Belgique, avec quelle ampleur les autorités d'occupation firent appel à des contributions informelles (collaborateurs, dénonciateurs, mouchards) et si le rôle des services de police belges reste vraiment, comme nos recherches préliminaires le donnent à penser, d'importance secondaire.

Ce n'est néanmoins pas un mince pari que de chercher à dégager ainsi des lignes de force, s'agissant de plusieurs milliers d'arrestations dont les victimes furent en majeure partie assassinées et n'ont jamais pu témoigner. La recherche internationale sur la Shoah n'offre pas d'exemples de pareille étude. À s'en tenir aux dossiers établis par les coupables, on peut trouver des sources extrêmement importantes sur I'arrestation d'isolés et de familles juives provenant de quelques services allemands. C'est par exemple le cas du Devisenschutzkommando (DSK) et des unités de gardes-frontière allemands en Belgique. Ces fonds d'archives sont néanmoins lacunaires et des dossiers de certains organes principaux de la répression ne subsistent que des débris épars, du fait que la Sicherheitspolizei, la F eldgendarmerie ou la justice militaire firent en grande partie disparaître leurs papiers dès avant la fin de la Seconde Guerre mondiale. D es centaines de documents ponctuels issus d'autres fonds, telles les archives de l'Association des J uifs en Belgique $(A J B)$, autorisent tout aussi peu à tirer des conclusions générales.

${ }^{1} \mathrm{Cfr}$. aussi pour ce qui suit : I. Meinen, «F ace à la traque. Comment les J uifs furent arrêtés en B el gique (1942-1944) », dans L es Cahiers de la M émoire contemporaine, nº 6, 2005, pp. 161-203. 
Mais il existe un autre accès à l'étude de la technique de persécution. Nous nous appuyons en premier lieu dans ce travail sur une recherche prosopographique partant des J uifs déportés. Ce faisant, nous mettons à contribution les sources suivantes: les dossiers d'avant-guerre de la Police des É trangers belge ; les fichiers, registres et listes de personnes établis par les autorités allemandes d'occupation ou à leur instigation aux fins de répression; en outre, des dossiers personnels que les autorités belges chargées des dédommagements établirent après 1945 ; ou encore des témoignages et procès-verbaux d'interrogatoires, menés par la police, de survivants, de proches de J uifs assassinés à Auschwitz, de sauveurs et témoins belges. Si I'on rapproche toutes les informations que l'on peut obtenir, on arrive à mettre à jour un matériel étonnamment abondant à propos des victimes de la Shoah. C'est vrai au premier chef de leur destin de persécutés antérieurement à 1942 et des conditions de leur arrestation.

L es sources se complètent mutuellement lorsqu'elles se rapportent à des personnes ou à des familles qui ont été appréhendées endéans un court laps de temps et ont été déportées ensemble par le même convoi. Une comparaison systématique des données portant sur les arrestations et les adresses officielles des J uifs déportés permet d'aller plus loin. L es deux approches amènent à concentrer sur un seul convoi les recherches prosopographiques. La question décisive est de savoir comment les forces allemandes d'occupation sont parvenues en un laps de temps relativement court et avec un personnel relativement restreint à interner à Malines des milliers de gens sans procéder à des rafles de grande envergure. Plus de 14.000 hommes, femmes et enfants juifs ont été arrêtés en dehors des grandes rafles et ne sont pas tombées aux mains de la Sicherheitspolizei allemande en conséquence d'« ordres de mise au travail » ou en provenance des camps de travail forcé du $N$ ord de la F rance. On a peine à imaginer que ces $14.000 \mathrm{~J}$ uifs furent tous arrêtés seuls ou avec leur famille. II faut donc chercher à établir si et avec quelle ampleur on en est venu à arrêter des groupes d'individus plus ou moins larges. Est-il possible de faire la preuve d'opérations ciblées d'arrestations menées à plusieurs reprises dans certains quartiers ou certaines rues, qui indiqueraient l'arrestation de J uifs à leur domicile officiel? 
Mais en fin de compte ce n'est pas de la technique utilisée par les persécuteurs qu'il est question dans cette étude, mais de l'histoire des J uifs déportés. Nous ne connaissons la plupart du temps leurs réactions face à la persécution et leurs efforts pour échapper aux représentants du régime nazi et à ses acolytes que grâce à des journaux et chroniques contemporaines des faits ou aux témoignages des rares survivants. Des recherches approfondies touchant un convoi déterminé peuvent en revanche mettre à jour un abondant matériel à propos de ceux qui ne sont pas revenus d'Auschwitz et dont les Allemands ont voulu effacer toute trace.

À ce jour, nous ne savons même pas au juste d'où venaient les J uifs déportés de Belgique. Dans quelles villes belges furent-ils signalés en dernier lieu ?2 Vivaient-ils déjà depuis pas mal de temps dans le pays ou ne s'y étaient-ils réfugiés que peu avant le début de la guerre? En provenance d'où et dans quelles circonstances les immigrants et réfugiés juifs - moins de $5 \%$ des victimes de la Shoah étaient citoyens belges - étaient-ils entrés en Belgique? Ceux qui avaient fui Allemagne après 1938 ou les Pays-Bas après 1942 en particulier avaient tenté, en prenant les plus grands risques, de se mettre en sécurité avant d'être finalement arrêtés. II vaut de plus la peine de rapprocher les stratégies individuelles de survie des futurs déportés en Belgique occupée - stratégies qu'on ne peut mettre au compte ni de la R ésistance, ni d'une contre-action juive organisées. Que peut-on découvrir touchant, par exemple, les formes de la clandestinité, les fausses identités ou les tentatives d'évasion vers la F rance?

\footnotetext{
$2 L$ aurence Schram a mené une recherche individuelle sur les adresses officielles sur base du fichier SD des convois IV à XI, en vue de déterminer le nombre des victimes des grandes rafles de l'été 1942. Cfr. M. Steinberg, La persécution des J uifs en Belgique (1940-1945), Bruxelles, 2004, passim; L. Schram, "L Les chiffres de la déportation de Malines à Auschwitz - perspectives et pistes », dans Musée juif de la Déportation et de la R ésistance à Malines (éd.), L 'expositi on belge à A uschwitz. Le livre, Malines, 2006, pp. 27-29. Pour la plupart des convois, les adresses officielles des J uifs déportés n'ont pas été relevées à ce jour. Lieven Saerens s'appuie dans son estimation statistique de la proportion des J uifs déportés par rapport à la population juive totale d'A nvers, de Bruxelles, etc. sur le fichier Beeckmans - AJ B, dans lequel les J uifs de Bruxelles sont néanmoins massivement sous-représentés. L es résultats obtenus par Saerens ne portent donc que sur $60 \%$ à peine des J uifs déportés de B elgique. Cfr. L. Saerens, « DeJ odenvervolging in B elgiëin cijfers », dans Cahiers d'H istoire du T emps présent, n 17, 2006, pp. 199-235.

3 Nous reprenons le concept de «stratégies individuelles de survie» à $A$. K riegel, «De la R ésistance juive », dans P ardès, n² 2, 1985, pp. 191-209, ici p. 202.
} 
Entre les tentatives de la population juive en vue de se mettre à l'abri et les procédés utilisés par les Allemands, il existait un rapport de réciprocité que Maxime Steinberg a de bonne heure indiqué. En prenant l'exemple du D evi senschutzkommando et des unités de la police allemande des frontières, on a pu montrer depuis lors que les tactiques mises en œuvre par les Allemands répondaient directement à la manière de faire des J uifs qui cherchaient au dernier moment encore à se soustraire à la déportation. Reste néanmoins à examiner si le passage de J uifs dans la clandestinité à partir de la fin de l'été 1942 fut réellement si massif qu'il exclut les grandes rafles et obligea l'occupant à procéder à des arrestations individuelles.

Tandis qu'on a beaucoup écrit sur le XX e convoi, dont plus de 230 J uifs s'échappèrent, nous ne savons que peu de choses du XXIe convoi ${ }^{4}$. Deux raisons motivent essentiellement notre décision d'entamer notre recherche par ce convoi de l'année 1943. II s'agissait de I'un des plus grands trains de la mort menant de B elgiqueà A uschwitz. Mais, dans les mois qui précédèrent son départ, on n'avait nullement assisté à une arrestation de masse. L e temps des grandes «J udenakti onen », lorsque les autorités d'occupation commencèrent par remettre des «ordres de mise au travail», puis avec l'appui de la police d'A nvers effectuèrent des rafles, pour enfin aller rechercher les hommes juifs dans les camps de travail forcé du Nord de la France, appartenaient déjà au passé à la fin 1942. Certes les Allemands avaient dès 1942 appréhendé individuellement ou en petits groupes près de $40 \%$ des J uifs déportés, mais depuis le début 1943 presque tous les convois à destination d'Auschwitz furent mis en route sans que de grandes rafles aient eu lieu5. À quoi s'ajoute que, pour le X X I e convoi, davantage de sources sont disponibles que pour la plupart des convois antérieurs. Ainsi, dans largement plus de 800 cas, des "reliques" nous

${ }^{4}$ Cfr. M. Steinberg, «Historique des convois», dans M émorial de la déportation des J uifs de B elgique, pp. 19-36, ici p. 31, ainsi que les statistiques qui y sont reproduites.

5 Pour les convois X II, X III et X IV de 1942 déjà, on ne dispose d'aucune indication d'arrestation de masse antérieure. Dans une recherche comparative de plus grande ampleur à laquelle nous travaillons, ces convois, ainsi que le X X Ve, de l'année 1944, seront pris en compte. L'importance de la part prise par les arrestations individuelles dans la déportation des J uifs en 1942 a été pour la première fois révélée dans: I. Meinen, " $F$ ace à la traque... », Les Cahiers de la $M$ émoire contemporaine, $n^{\circ} 6,2005$, p. 162 sqq. II faut par ailleurs prendre en compte les chiffres publiés entre-temps par L aurence Schram : cfr. Musée juif de la Déportation et de la R ésistance à Malines (éd.), L'exposition belge à A uschwitz..., Malines, 2006, p. 28 
ont été transmises, donc des documents personnels confisqués par la Sicherhei tspolizei, que les J uifs portaient sur eux lorsqu'ils furent appréhendés, parmi lesquels de fausses cartes d'identité ou des documents d'émigration.

L e X X I e convoi partit le 31 juillet 1943 de Malines avec à son bord $1.560 \mathrm{~J} \mathrm{uifs}^{6}$. Dans le train se trouvaient 244 enfants et adolescents de moins de 18 ans; dans ce nombre, 105 garçons et 69 filles âgés de moins de 15 ans, dont 62 n'avaient pas encore atteint leur sixième année. Parmi les tout-petits, 14 nourrissons qui furent presque tous placés sur les listes de déportation avec leur mère en cette année 1943 qui les avait vus naître. Une part prépondérante des déportés (75\%) était constituée d'hommes et femmes adultes, dont l'âge allait de 18 à 59 ans. L'âge de $151 \mathrm{~J}$ uifs oscillait entre 60 et 80 ans. Une femme avait 84 ans accomplis. 849 personnes possédaient la nationalité polonaise, 285 la nationalité allemande ou ex-autrichienne, 35 la nationalité extchécoslovaque; 97 des déportés étaient des Néerlandais, 23 des B elges ; 131 personnes étaient considérés comme apatrides ; 99 étaient de nationalité indéterminée. $57 \mathrm{~J}$ uifs s'étaient enfuis de convois précédents à destination d'A uschwitz, dont 41 du XX e. 6 hommes et 2 femmes au moins prirent le risque de s'échapper du XXIe convoi. 5 d'entre eux y réussirent et échappèrent à une nouvelle arrestation ; 3 tombèrent sous les balles des militaires de l'escorte. Un autre homme fut abattu dans des circonstances non expliquées ; peut-être s'agissaitil également d'une tentative d'évasion.

Pour surveiller les trains partant de Belgique jusqu'à la frontière allemande, I'Office central de sécurité du Reich (Reichssicherheitshauptamt, RSHA) désignait normalement des détachements de I'Ordnungspolizei, qui venaient à chaque fois d'Allemagne avant le départ du convoi. A près l'évasion massive de J uifs du X X e convoi - en sus ou en remplacement -, un détachement de la SS-W achkompanie de l'avenue L ouise les accompagna?.

\footnotetext{
${ }^{6}$ Les données de base que nous avons pu établir divergent légèrement des informations qu'on trouve dans le $M$ émorial de la déportation des J uifs de Belgique..., dans L'exposition belge à A uschwitz..., et chez D. Czech, $K$ alendarium der $E$ reignisse im $K$ onzentrationslager A uschwitzBirkenau 1939-1945, R einbek b. H amburg, 1989, p. 562.

7 L es postes de commandement de la W achkompanie étaient occupés par des SS allemands, tandis que les équipes se composaient de volontaires flamands, wallons, et sans doute aussi hongrois et roumains. Parmi les SS allemands, I'accompagnement des trains de la mort était
} 
Le 2 août 1943, deux jours après son départ, le train parvint à Auschwitz. Nous ignorons combien de gens étaient morts en route. Compte non tenu de cette inconnue, nous devons supposer que 1.085 J uifs furent gazés dès leur arrivée. Parmi les victimes assassinées se trouvaient 565 femmes et 520 hommes, bébés et jeunes enfants compris. $466 \mathrm{~J}$ uifs, dont 211 femmes, furent sélectionnés pour le travail et reçurent un matricule de détenu. Une grande partie des hommes arriva au commando de J aworzno, qui n'avait été créé que peu auparavant, où ils travaillèrent comme esclaves dans les mines de charbon. Sur les $1.560 \mathrm{~J}$ uifs qui partirent du camp de Malines par le X X I convoi, il ne restait plus en vie en 1945 - indépendamment des évadés - que 42 hommes et femmes. Parmi eux, Itta WIERNIK, qui dut jouer du violon dans le M ädchenorchester d'Auschwitz, et Sarah GoldbeR G, "pianiste" de l'"Orchestre rouge" et membre des Partisans armés de $B$ ruxelles.

L e martyre des J uifs commença avec l'arrivée du train à A uschwitz. E t pourtant, notre étude doit se limiter à sa préhistoire. En son centre figurent des hommes, des femmes et des enfants juifs du XX I e convoi dont les biographies ne peuvent évidemment être rendues que par extraits. L'histoire de leur vie donne à voir non seulement les étapes d'une persécution en voie de radicalisation permanente, mais aussi leurs efforts désespérés pour se protéger eux-mêmes ou les leurs contre l'emprise des Allemands et la déportation. La première partie de l'essai, organisé chronologiquement, qu'on lira ci-après se consacre aux multiples aspects de la fuite des J uifs au temps de la persécution et de la déportation entre 1938 et 1943. II y est d'abord question de ces réfugiés qui parvinrent en Belgique après 1938 en provenance des territoires sous domination allemande et de leurs conditions de vie avant et après l'Occupation. Nous parlerons de surcroît d'hommes et de femmes qui, après l'invasion de la W ehrmacht et dans les années 1942-1943 encore, alors que les trains se rendaient déjà à Auschwitz, tentèrent de fuir vers la $\mathrm{F}$ rance, la $\mathrm{B}$ elgique et les $\mathrm{P}$ ays-B as occupés. $\mathrm{A}$ la présentation biographique s'ajouteront dans la seconde partie des considérations statistiques et une synthèse historique générale. Pour

«particulièrement apprécié » en raison des avantages matériels qui y étaient liés ; interrogatoire Böhlich, 23.4.1968, Zentrale Stelle der L andesjustizverwaltungen L udwigsburg, AR -Z 18/61, Bd. 37, BI. 308-313. 
une meilleure compréhension, il faut néanmoins anticiper ici un résultat quantitatif des recherches prosopographiques. Si, dans ce qui suit, ce n'est dans la plupart des cas pas A nvers, mais B ruxelles qui est indiqué comme adresse officielle ou lieu d'arrestation, cela correspond au fait que ce sont majoritairement des J uifs de Bruxelles qui se trouvaient dans le XX I e convoi. Plus de 1.200 des 1.560 hommes, femmes et enfants déportés au total étaient enregistrés dans l'agglomération bruxelloise, moins de 160 dans le Grand-A nvers.

\section{9) \\ Réfugiés d'Autriche, de Tchécoslovaquie et d'Allemagne (1938-}

Fin mai 1938, Max Gottschalk, vice-président de la Communauté israélite de B ruxelles et président du Comité d'A ide et d'A ssistance aux Victimes de I'A ntisémitisme en Allemagne (CAAVAA) fondé en 19338, transmit comme convenu à l'administrateur de la Sûreté publique, responsable de la Police des É trangers belge, une liste nominative des réfugiés juifs confiés aux bons soins du Comité 9 . La liste comprenait approximativement 250 personnes, presque exclusivement des hommes jeunes ou d'âge moyen, surtout des réfugiés d'origine autrichienne, qui avaient fui leur pays au printemps 1938, immédiatement après I'annexion (A nschluss) de l'Autriche au Reich allemand et les pogroms antijuifs. S'y ajoutaient des ressortissants polonais et des apatrides. Comme les J uifs qui parvenaient en Belgique, souvent par des voies illégales, ne se signalaient pas toujours auprès d'institutions officielles ou caritatives et s' efforçaient d'assurer leur existence de leurs propres deniers ou en acceptant des travaux d'occasion, le chiffre de ceux qui parvinrent dans le pays avec la première vague de réfugiés de l'année 1938 est à coup sûr considérablement plus élevé que cette liste ne le donne à entendre ${ }^{10}$. De plus, nombre d'entre eux ne gagnèrent pas B ruxelles, mais A nvers, pour s'y préparer à poursuivre leur voyage.

\footnotetext{
${ }^{8} \mathrm{Cfr}$. aussi pour ce qui suit : J .-Ph. Schreiber, « L'accueil des réfugiés juifs du $R$ eich en $B$ elgique. Mars 1933 - septembre 1939 : le Comité d'Aide et d'A ssistance aux Victimes de l'A ntisémitisme en Allemagne », dans L es Cahiers de la M émoire contemporaine, $n^{\circ} 3,2001$, pp. 23-71.

${ }^{9}$ Archives générales du R oyaume ( $A G R$ ), Dossiers généraux de la Police des Étrangers (2e versement), dos. 818.

10 L e nombre de réfugiés juifs présents en B elgique en 1939-1940 est estimé par différentes sources à 25.000 ; voir la deuxième partie de cette étude.
} 
Les autorités belges, tout comme les organisations caritatives juives et sans doute la plupart des réfugiés eux-mêmes, ne considéraient la Belgique que comme un pays de transit, étape préalable à une émigration vers l'outre-A tlantique ou la Palestine. N éanmoins, comme les pays d'accueil n'étaient pas légion ou que les visas ou documents de voyage nécessaires ne purent plus être délivrés dans les délais, bien des gens qui avaient encore espéré pouvoir prendre le bateau restèrent bloqués à Bruxelles ou dans les quartiers juifs d'Anvers jusqu'au moment où les Allemands attaquèrent le pays. Ainsi, la fuite d'A utriche représenta pour au moins 46 des personnes portées sur la liste du Comité de B ruxelles le début de l'histoire d'une persécution qui devait s'achever sous l'occupation allemande de la Belgique par la déportation à Auschwitz. 9 des hommes et femmes mentionnés se retrouvent sur les listes, établies au camp de transit de Malines, du convoi $n^{\circ}$ XX I du 31 juillet 1943. Parmi eux I sraël Dienstag (né à Bursztyn en Pologne, venant de Vienne, 27 ans, vulcanisateur), Arthur H OLZER (né à Vienne, 33 ans, typographe, dont la famille se trouvait encore en Autriche) et Arthur Sonnenschein (né à Vienne en 1915, coiffeur).

Les parents d'Arthur Holzer, son père, sa mère et ses deux frères, s'exilèrent d'Autriche dès l'année 1938, et la femme de H olzer, Cäcilie SCH USTER, née à V ienne en 1905, couturière, réussit, en 1939 au plus tard, à gagner Bruxelles au départ de Vienne en compagnie de sa fille de 8 ans, E rika. La famille reçut un permis de séjour, qui devait être reconduit tous les six mois, et habita de 1941 au début 1943 à diverses adresses dans la commune d'Anderlecht, l'un des centres de l'immigration juive en provenance d'E urope centrale et orientale. H olzer, imprimeur de formation, travaillait maintenant aussi comme tailleur. Des autres conditions de vie personnelle de la famille en Belgique occupée par les Allemands, nous ne connaissons - indépendamment de la situation oppressante à laquelle tous les J uifs étaient exposés depuis la publication à partir de l'automne 1940 des ordonnances antijuives pour ainsi dire rien. Sur les circonstances de leur arrestation, on ne dispose que de quelques indications. Le nom d'Arthur Holzer figure d'abord sur la liste de déportation du convoi $n^{\circ}$ X VIII du 15 janvier 1943. Ou bien il fut ajourné pour des raisons impossibles à préciser, ou bien, plus vraisemblablement, il s'échappa durant le transfert et fut 
repris trois mois plus tard, peut-être avec sa femme et son enfant. Tous trois furent en tout cas assignés au convoi $n^{\circ} X X$, en partance le 19 avril 1943, duquel, avec l'appui de résistants, une évasion massive réussit. $\mathrm{H}$ olzer put prendre la fuite, tandis que Cäcilie $\mathrm{H}$ olzer-Schuster et sa fille étaient emmenées à Auschwitz. Mais Holzer lui-même ne devait pas échapper aux Allemands. II fut réincarcéré à Malines fin avril, placé sur une "liste spéciale" des personnes échappées du XX e convoi et finalement déporté avec le $X X \mid$ e, dans lequel se trouvaient aussi ses parents Sigmund et $\mathrm{K}$ athy $\mathrm{H}$ olzer, née Singer, et son frère Maximilien, arrêtés fin juin 1943. On ne peut établir que les arrestations se soient déroulées conjointement, mais ce n'est pas invraisemblable. À l'exception du plus jeune frère, Wilhelm Holzer, aucun membre de cette famille n'a survécu.

L'aide-coiffeur Arthur SONNENSCHEIN, qui avait quitté en 1938, à 23 ans, sa ville natale de $V$ ienne et figurait lui également sur la liste des réfugiés d'Autriche commis aux bons soins du comité d'aide juif de B ruxelles, semble, dès I'offensive de l'armée allemande en mai 1940, avoir cherché à gagner au plus vite la France. Fin août, il rentra néanmoins de L ille dans la capitale belge, où il est mentionné sous son vrai nom au printemps 1942. II se procura peu après un faux passeport au nom d'H enri Vandeperre, qui le donnait pour citoyen belge. À la mi-juin 1943, il fut pourtant arrêté et identifié comme juif. Comme presque toutes les victimes appréhendées à B ruxelles, il fut sans doute d'abord emmené à l'avenue L ouise, siège du service des Affaires juives de la Gestapo en Belgique. Quelques jours plus tard, il fut transféré avec d'autres détenus au camp de Malines. On lui enleva ses papiers, dont son faux passeport, qui a été conservé. II porte en allemand la surcharge "J uif, faux », de la main d'un des sbires de la SS. A rthur Sonnenschein ne parvint pas à A uschwitz. Selon une information due à un homme également déporté par le $X X \mid$ e convoi, mais qui survécut aux camps de la mort, il fut abattu au cours du voyage vers l'Est. Nous ne pouvons savoir s'il paya de sa vie une tentative d'évasion ou fut autrement la victime de l'arbitraire meurtrier de ses gardiens.

La "crise des Sudètes" et l'annexion subséquente de parties de la Tchécoslovaquie par le Reich allemand amena dans la seconde moitié de l'année 1938 une nouvelle vague de réfugiés en Belgique. Dix au moins de ces réfugiés de T chécoslovaquie se retrouvent sur les listes de 
déportation du 31 juillet 1943. Parmi eux, Hers GR OSS, un enseignant né en 1899, originaire de Vulchovce (localité située dans une région frontalière de Transcarpatie autrefois austro-hongroise). II avait cherché refuge à Anvers mais, après l'occupation de la B elgique par la W ehrmacht, il fut expulsé, début 1941, vers la province du Limbourg. Cette mesure de contrainte, sur laquelle on reviendra, eut lieu sur ordre de l'administration militaire allemande et affecta un large groupe de J uifs étrangers. À la fin de l'été, Gross put se rendre à B ruxelles, où il travailla à partir de 1942 au plus tard comme bedeau de la synagogue et professeur de yiddish à la Communauté israélite qui, en mars 1943 encore, attestait que son travail était indispensable. II vira pour la dernière fois en avril 1943 une part de son modeste salaire à sa femme, qui était restée dans cette partie de la Slovaquie entretemps réannexée par la H ongrie. Lorsqu'un mois plus tard il fut arrêté, les Allemands eurent des difficultés à déterminer la nationalité de Gross, parce que lui aussi possédait un permis de séjour pour la Hongrie. La nationalité hongroise l'aurait peut-être à cette époque préservé de la déportation, mais $\mathrm{H}$ ers Gross était citoyen tchécoslovaque et, comme tous les J uifs déportés de Belgique, il fut sans autre forme de procès déclaré apatride, avant de partir avec le X X I e convoi.

Nathan SILBERMANN, de K ošice, né en 1892, son épouse du même âge, H elene née POSTR ONG, originaire de Dukla, et leurs cinq enfants détenaient également la nationalité tchécoslovaque. Mais la famille s'était installée dès le lendemain de la Première Guerre mondiale à Cologne, où les enfants étaient nés entre 1922 et 1930. Silbermann travaillait comme marchand et représentant. Helene Postrong resta femme au foyer. Les deux filles aînées, J ohanna et Gerda, entrèrent en apprentissage respectivement comme couturière et gérante. $\mathrm{F}$ in 1938 ou début 1939, peu après le pogrom de novembre, qui déclencha la troisième grande vague de réfugiés en quelques mois, les parents s'exilèrent de Cologne en franchissant manifestement de manière légale la frontière belge, à la différence de la majorité des réfugiés juifs à cette époque. Les enfants arrivèrent en B elgique séparément et avec l'appui d'œuvres d'entraide juives. Comme des milliers d'autres J uifs sans moyens, la famille s'établit près de la gare du Midi dans la commune bruxelloise de Saint-Gilles. Début 1942, Nathan Silbermann se retrouva aux prises avec le D evisenschutzkommando de B ruxelles, l'une 
des antennes du ministère des Finances du Reich spécialisé dans la recherche de devises, mais aussi, de plus en plus, dans la persécution des J uifs. On peut relever dans une note du secrétaire aux douanes B., responsable de ce type d'enquêtes, que le bureau du DSK à B ruxelles avait appris «confidentiellement »- c'est-à-dire par un informateur travaillant pour les Allemands - «que le J uif Silbermann, domicilié à Bruxelles, rue $V$ an Helmont 6, serait en possession de sommes considérables en dollars et en livres britanniques. De plus, Silbermann se livrerait au trafic de devises. » II était en outre spécifié que Silbermann, arrivé trois ans plus tôt en Belgique «comme un mendiant », serait à présent « un homme aisé ». A près une perquisition par des membres du DSK, Silbermann se vit ordonner de se présenter pour «traitement » de son cas au bureau de la rue de la L oi. II tenta alors de rendre compte de la provenance de l'argent étranger qui avait été trouvé sur lui et confisqué. II explicita sa propre situation matérielle, qui jette une lumière sur celle de beaucoup d'autres réfugiés juifs en B el gique, mais aussi sur I'hospitalité du pays, qui se perpétuait en dépit des dures conditions de l'occupation allemande: «Pour ma part, j'ai fui il y a trois ans l'Allemagne (Cologne) [... ] pour B ruxelles. Mon épouse et moi avions un visa pour entrer en Belgique, tandis que mes cinq enfants sont arrivés en Belgique par l'intermédiaire du Comité des Enfants. En Allemagne, j'étais représentant en produits textiles. J e les achetais de mes propres deniers à des grossistes, pour les vendre aux consommateurs. J e n'avais pas de fortune. À mon entrée en Belgique je n'ai emporté de somme d'argent d'aucune sorte. Mon mobilier, ma garde-robe et mon linge ont été emmenés régulièrement en Belgique. Depuis trois ans que je réside en Belgique, je n'ai eu aucune occupation professionnelle. Je touche depuis ce temps le secours aux nécessiteux de la Ville de Bruxelles. Cette aide se monte actuellement à 270 francs belges par semaine. De plus, je reçois des dons ponctuels, comme du charbon, du pain et d'autres denrées alimentaires. $\gg^{11}$

Qu'en dépit de sa détresse matérielle, il ait pu effectuer quelques modestes versements pour aider des parents en Europe orientale s'expliquait, dit-il, par la vente d'effets personnels. Le DSK y vit « une

11 DSK, dossier N athan Silbermann, 7.1.1942, CE GE S, AA 585/53/8. 
excuse typiquement juive » et veilla à ce que $\mathrm{N}$ athan Silbermann soit condamné à une amende et ne récupère pas une caution qu'il avait déposée. Le destin ultérieur de la famille n'est plus que maigrement documenté dans les archives. Les filles, J ohanna et Gerda, furent déportées par l'un des premiers convois en partance de Malines, le 15 août 1942. Helene Silbermann-Postrong et sa fille Margot, née à Cologne en 1929, vivaient sans doute cachées lorsque, fin juin 1943, elles furent appréhendées dans la commune d'E tterbeek - donc pas à leur adresse officielle. Elles se trouvaient dans le convoi de déportation $n^{\circ} X X I$ et furent assassinées à Auschwitz. Nathan Silbermann survécut à l'occupation en Belgique.

\section{L'échange d'enfants entre les Pays-Bas et la Belgique}

Les pogroms des 9 et 10 novembre 1938 et l'exode massif de J uifs d'Allemagne réduisirent définitivement à l'absurde la politique d'accueil des réfugiés de la B elgique et des autres É tats voisins occidentaux, qui restait déterminée par le concept traditionnel de réfugié politique et n'intégrait pas le fait d'un groupe de population menacé dans son existence physique. L'indice sans doute le plus marquant en fut l'arrivée en B elgique d'enfants juifs non accompagnés, qui avaient été placés par leurs parents ou connaissances dans l'express CologneAix-la-Chapelle-Bruxelles dans l'espoir qu'ils seraient accueillis et aidés. IIs ne portaient quelquefois sur eux ni papiers ni argent, mais seulement l'adresse d'un point de chute à B ruxelles ou à Anvers. La police allemande des frontières laissa passer les enfants, intéressée qu'elle était de toute façon à voir sortir du pays autant de J uifs que possible, au mépris des accords conclus et en éludant les contrôles frontaliers belges. À H erbesthal, alors gare frontalière belge, la CroixRouge recueillit ces réfugiés mineurs mais, dans plusieurs cas documentés, la Police des É trangers de B ruxelles les traita comme les adultes parvenus illégalement dans la zone frontière et les expulsa vers l'Allemagne. Pareil événement - le refoulement d'un convoi de 35 enfants - causa au début janvier 1939 un scandale politique en B elgique, souleva une vague de solidarité spontanée dans tout le pays et entraîna un assouplissement momentané de la politique belge d'asile, au demeurant peu cohérente, et dès lors plutôt à l'avantage des 
réfugiés d'Allemagne nazie12. Le gouvernement belge se déclara notamment prêt à accueillir des contingents d'enfants juifs et plusieurs institutions caritatives s'employèrent à héberger ces enfants dans des familles ou des homesis.

L'une de ces organisations privées, le Comité d'Assistance aux Enfants juifs réfugiés (CAEJ R), fondé à Bruxelles en novembre 1938, intervint également par la suite dans la mise sur pied d'un échange d'enfants entre les Pays-Bas et la Belgique visant à réunir à leurs parents réfugiés en Belgique des enfants juifs d'Allemagne et d'Autriche qui, arrivés seuls en $\mathrm{H}$ ollande, y avaient été hébergés dans des homes. Des cas de même nature dans le pays voisin trouvaient une solution parallèle. C'est ainsi que $\mathrm{H}$ ermann Gold, 6 ans à l'époque, put quitter Amsterdam et, en avril 1940, trois semaines avant que l'armée allemande ne fasse mouvement vers la Hollande et la Belgique, retrouver ses parents, qui avaient réussi en janvier 1939 à fuir Duisburg pour gagner Anvers ${ }^{14}$. Leibus GOLD et B ajla BIR MAN étaient tous deux nés en Pologne en 1897, s'étaient établis en Allemagne après la P remière Guerre mondiale et étaient tenus dès 1935 pour apatrides. Gold était de formation peintre en bâtiment, mais il gagnait manifestement de quoi faire vivre les cinq personnes de sa famille comme marchand de fruits dans la ville industrielle rhénane de Duisburg. C'est tout de suite après le pogrom de novembre que la famille doit avoir décidé d'émigrer car, début décembre 1938, le bureau des D evises de I'Oberfinanzpräsi dent de Düsseldorf l'autorisa à exporter son mobilier. Nous ignorons le destin des deux plus âgés des enfants.

\footnotetext{
12 Voir les documents et la collection de coupures de presse de cette période dans AGR, D ossiers généraux de la Police des Étrangers (2e versement), dos. 793 («Exode vers la B elgique d'enfants juifs provenant d'Allemagne »).

13 En 1940, sous l'occupation allemande, des groupes entiers de ces enfants furent renvoyés en territoire du R eich ; voir p. ex. rapport Gendarmerie nationale, «Entrée des enfants israélites en B elgique », 24.6.1939 ; note Sûreté publique et «L iste nominative des enfants venant de Vienne par les soins du Comité des Avocates de Belgique», 7.9.1940, AGR, Dossiers généraux de la Police des Étrangers (2e versement), dos. 793. Cfr. M. Ansbacher, « R escue and R eturn : Post$K$ ristallnacht German-J ewish R efugee Children in Belgium and their R eturn to Germany in 1941 », dans D. Michman (É d.), B elgium and the H olocaust. J ews, B elgians, Germans, J érusalem, 1998, pp. 433-443, ainsi que S. Collignon, «Les homes Bernheim et Speyer (1938-1940). Témoignages d'enfants réfugiés d'Allemagne et d'Autriche», dans Les Cahiers de la M émoire contemporaine, $n^{\circ} 6,2005$, pp. 21-67.

${ }^{14}$ Lettre CAEJ R des 16.2.1940 et 19.4.1940; note Sûreté publique, 21.2.1940, AGR, Dossiers généraux de la Police des É trangers (2e versement), dos. 793.
} 
H ermann Gold fut expédié aux Pays-Bas. L es parents franchirent la frontière belge. Le 20 janvier 1939, ils se firent enregistrer à Anvers, vraisemblablement pour régler la suite de leur voyage. Commença alors une période de perpétuels changements d'adresse, d'abord à A nvers même. C'est là qu'en décembre 1940 la famille - H ermann Gold était entretemps arrivé en B elgique - fut inscrite au R egistre des J uifs, dont la tenue avait été imposée par l'occupant. Temporairement déportés, comme beaucoup de J uifs, dans la province du Limbourg, B ajla Gold-Birmann et son fils purent quitter dans la seconde moitié de 1941 leur résidence forcée de Zutendaal au L imbourg pour regagner Anvers, tandis que Leibus Gold était envoyé dans la capitale, où il se chercha d'abord des logements successifs à Schaerbeek, troisième commune bruxelloise en importance par sa population juive. A pparemment, la famille ne parvint pas à se réunir pendant tout un temps. Le 5 juin 1943 encore, Gold fit prolonger de six mois auprès des autorités bruxelloises compétentes sa carte d'identité pour étranger, muni de la surcharge tamponnée en rouge «J uif - J ood»; sa femme resta inscrite officiellement à Anvers. À cette date au plus tard, tous deux plongèrent avec leur jeune fils dans la clandestinité. Comme tant d'autres J uifs persécutés, ils trouvèrent refuge dans une famille belge qu'ils connaissaient à B ruxelles. Début juillet 1943, tous trois y furent arrêtés et emmenés à la caserne Dossin à Malines. Le nouveau commandant du camp, Frank, avait permis, pour que l'entretien des internés dépende d'envois privés, de recevoir des colis de vêtements et de vivres. C'est ainsi que ses logeurs de B ruxelles s'occupèrent jusqu'au 27 juillet d'approvisionner la famille Gold. Quatre jours plus tard, Hermann Gold, à présent âgé de 9 ans, partait pour la mort avec ses parents.

Ce garçon n'était pas le seul dans le $X X \mid$ e convoi à être venu en B elgique via l'échange d'enfants avec les Pays-B as. J osef SI LBERBERG aussi, avec sa sœur cadette Cilli, fut proposé en octobre 1939 pour un échange similaire par le Comité d'Assistance aux Enfants juifs réfugiés ${ }^{15}$. Il avait alors 16 ans et était hébergé au $K$ indercomité, H eerengracht à Amsterdam. Ses parents, des apatrides originaires de Pologne, avaient fui en décembre 1938 la région d'Aix-la-Chapelle et

15 Lettre CAEJ R des 9.10. et 26.10.1939, AGR, Dossiers généraux de la Police des Étrangers (2e versement), dos. 793. 
habitaient Anvers-Berchem. En 1942, J osef résida un moment chez son frère aîné de quatre ans, $\mathrm{H}$ erman, à B ruxelles. Celui-ci fut déporté de Belgique dès août 1942 par le convoi III. Le cadet survécut une petite année, sans doute illégalement, avant d'être arrêté et, fin juillet 1943, incarcéré à Malines.

\section{Passagers du St-Louis}

L'errance, en mai 1939, du St-L ouis, un paquebot de la ligne Hambourg-Amérique, et la tragédie de ses passagers juifs, qui quittèrent légalement l'Allemagne, mais à qui on refusa le débarquement à Cuba au prétexte que leurs visas n'étaient pas valables car achetés au prix fort à un fonctionnaire cubain, a été souvent décrite. On sait moins que la B elgique - après que les É tatsUnis eussent également refusé l'entrée sur leur territoire - fut le premier É tat européen à se déclarer prêt à accueillir une partie des réfugiés, et ce à l'intervention de l'influent président du Comité d'aide de Bruxelles, Max Gottschalk déjà citéit. Le bateau remit le cap sur I'Europe et, le 17 juin, entra dans le port d'Anvers. La Belgique accueillit plus de 200 passagers. Le couple Ludwig BEN DHEIM - Berta SCHLESSINGER, qui était du nombre, conserva son billet de première classe Hambourg-La Havane, qui avait coûté près de 2.000 Re ichsmark, et le permis d'embarquer établi par le Comité d'A ssistance aux R éfugiés juifs (I'organisation qui succéda au CAAVAA) et portant le tampon des autorités du port d'Anvers, jusqu'à ce qu'ils leur soient retirés au camp de Malines en 1943.

Berta Schlessinger et $L$ udwig Bendheim étaient nés tous deux à F rankfort-sur-le-Main. Lorsqu'ils avaient décidé d'émigrer à Cuba et de là sans doute aux États-U nis, B endheim, un ancien commerçant, avait déjà 65 ans. Durant la Première Guerre mondiale, il avait exercé au tribunal cantonal allemand ( $K$ aiserliches $D$ eutsches B ezirksgericht) de Charleroi les fonctions d'interprète et d'auxiliaire administratif. En 1935 encore, il s'était vu décorer de la « Croix d'honneur des anciens combattants » (E hrenkreuz für $\mathrm{F}$ rontkämpfer) créée par le président du

\footnotetext{
16 Cfr. B. Garfinkels, B elgique, terre d'accueil, B ruxelles 1974, pp. 149 sqq. ; F. Caestecker, Ongewenste gasten. J oodse vluchtelingen en migranten in de dertiger jaren, B ruxelles, 1993, pp. 245 sqq.
} 
$\mathrm{R}$ eich $\mathrm{H}$ indenburg. II se retrouvait à présent avec sa femme, sa cadette de plus de dix ans, couturière de formation, comme réfugié dans le pays, qui devait être peu après une nouvelle fois occupé par les Allemands. Tous deux cherchèrent à échapper à l'offensive de la W ehrmacht en mai 1940 en prenant la direction de la F rance, au beau milieu de la cohue d'innombrables fugitifs. Peu avant Dunkerque, dans la ville balnéaire de La Panne en Flandre occidentale, ils se retrouvèrent pris entre les deux fronts, furent momentanément incarcérés, puis - rattrapés par les chars allemands - renvoyés à B ruxelles. Fin 1940, ils s'y firent inscrire au R egistre des J uifs. A près avoir à plusieurs reprises changé de domicile, ils habitèrent finalement le quartier résidentiel de Watermael-Boitsfort, avant de trouver une cachette. L e 29 avril 1943, des gestapistes effectuèrent une perquisition chez une Belge membre de la Résistance. À cette occasion, ils découvrirent le couple Bendheim et une autre femme juive, qui s'était également cachée. Après avoir été détenus plusieurs semaines à la prison militaire de Saint-Gilles, réquisitionnée par les forces d'occupation, L udwig Bendheim et Berta Schlessinger furent placés le 5 juin sur les listes du X X I e convoi en partance de Malines et envoyés à la mort le 31 juillet 1943.

C'est par le même train de déportés que Gerda HERMANN et sa mère Sofie K LEIN-HERMANN allèrent à Auschwitz. Natives de Berlin, elles avaient résidé jusqu'en 1939 à Munich. Avec le mari, J ulius H ermann, décédé en 1941, elles aussi étaient rentrées en E urope avec le St-Louis'17. Nous ne pouvons dire au milieu de quels espoirs et de quelles angoisses le couple Bendheim et la famille Hermann avaient passé les années 1940 à 1943 dans la Belgique sous occupation allemande. $E n$ tout cas, beaucoup de réfugiés étaient conscients d'un danger imminent. Quant, à l'été 1942, les premiers « ordres de mise au travail » furent envoyés, qui faisaient obligation aux J uifs de se présenter pour le transfert vers l'E st, Gerda H ermann, retraitée depuis les années 30, se fit préventivement établir par le bureau de l'emploi à B ruxelles un certificat attestant sa totale incapacité à travailler. II ressort cependant aussi des papiers personnels qui lui furent confisqués lors de sa déportation qu'elle conserva son abonnement hebdomadaire

17 Voir D SK, dossier J ulius H ermann, 19.11.1940, CE GE S, AA 585/52/4. 
aux tramways municipaux de Munich, comme si elle avait encore pu avoir à l'utiliser, tandis qu'elle et sa mère, fin septembre 1942, devaient remettre, sur ordre de l'Oberfeldkommandantur de B ruxelles, les passeports dont elles excipaient en tant que citoyennes allemandes.

\section{F aux visas et fuite organisée en 1939-1940}

L es archives de la Sûreté publique de Bruxelles des années 19391940 sont pleines de notes touchant l'actif commerce de visas de transit ou d'entrée falsifiés ou établis par complaisance par des représentants diplomatiques belges ou d'outre-Atlantique - des pratiques dont bon nombre de Juifs d'Autriche et d'Allemagne furent les victimes, mais auxquelles au moins autant durent la vie ${ }^{18}$. La même considération vaut pour ce qui est de l'aide rémunérée à la fuite.

E $n$ avril 1940, un réfugié allemand de 30 ans du nom d'E rich BIER attira l'attention de la police de Bruxelles. Bier, qui aurait exercé le métier de professeur delangues, mais aussi d'imprimeur, était arrivé de Cologne en Belgique avec sa famille l'année précédente. Manifestement, quelqu'un l'avait donné, car on peut lire dans le rapport du commissariat de police qu'un quidam avait estimé « que le Dr. R. Bier [sic], domicilié à Saint-Gilles, 35 avenue du R oi, et un certain S. [... ], que I'on peut atteindre tous les jours vers 13 heures par téléphone, promettent, moyennant un montant minimum de 3.500 francs belges, d'aider des J uifs allemands à émigrer d'Allemagne dans notre pays $\gg 19$. Si la dénonciation reposait sur des faits reste incertain. On ne peut non plus dire si Bier, qui dispensait à d'autres J uifs des cours d'anglais, s'était réellement prévalu d'un titre de docteur falsifié. Toujours est-il que le prix indiqué, qui devait payer l'entrée illégale sur le territoire et rémunérer divers complices, n'était pas inhabituellement élevé. E n mai 1940, B ier fut temporairement expulsé vers la France, avec des milliers $\mathrm{d}^{\prime}$ « étrangers indésirables », et interné, mais il put bientôt regagner B ruxelles. À partir de fin 1941, il obtient un emploi comme garçon ou aide-cuisinier dans un hôtel de la gare du Nord réquisitionné par les

\footnotetext{
18 V oir D ossiers généraux de la Police des Étrangers (2e versement), dos. 35 («F aux visas ou visas délivrés abusivement par les postes consulaires de B elgique à l'étranger »).

${ }^{19} \mathrm{R}$ apport du 11.4.1940, AGR, Dossiers généraux de la Police des Étrangers (2e versement), dos. 35.
} 
Allemands pour servir de «foyer des artistes ». Ce qui conduisit après la guerre, longtemps après qu'il eut été assassiné par les Allemands, à le soupçonner d'avoir collaboré avec l'occupant. On nous apprend il est vrai que Bier donna à son second fils, né en août 1941, le prénom patriotique de Leopold. En outre, dans des circonstances peu claires, peut-être sur base d'une dénonciation ou suite à une empoignade avec un soldat de la W ehrmacht, il fut arrêté en avril 1942 sur son lieu de travail et placé en détention. Tandis que le reproche d'avoir dérobé des timbres de ravitaillement était abandonné, le tribunal de la F eldkommandantur de Bruxelles le condamna en juin 1942 pour infraction aux dispositions antijuives à une année de prison, parce qu'il ne s'était pas fait inscrire, comme il le fallait, au R egistre des J uifs, qu'il avait utilisé un faux nom et n'avait pas observé le couvrefeu en vigueur pour les J uifs. Cette sentence émanant d'un juge militaire allemand équivalait à une condamnation à mort. De 1942 au début de l'été 1943, E rich Bier purgea sa peine à la prison de Saint-Gilles et au camp de réfugiés de Merksplas, utilisé par l'occupant. En juin 1943, on I'amena à Malines, d'où il fut déporté par le X X I e convoi. Sa femme et ses deux fils ont survécu à la persécution.

E n ce qui concerne l'aide organisée, rémunérée, à la fuite des I uifs d'Allemagne vers la Belgique, quelques cas spectaculaires sont bien documentés, parce qu'ils débouchèrent en Allemagne sur des procès criminel $\mathrm{s}^{20}$. Les autorités belges enquêtèrent à partir de mai 1939 sur une entreprise de ce genre, à laquelle on reprochait son «trafic de réfugiés juifs » et dont on soupçonna un tailleur juif d'origine polonaise et habitant à Anvers du nom de Sch. d'être le «chef ». La «centrale » de ce groupe de près de vingt personnes, qui se composait prétendument de recruteurs professionnels de J uifs en Allemagne, d'un chauffeur stationné à E upen-Malmedy, de " passeurs », de faussaires et d'un «banquier », était censée se trouver dans un café d'Anvers et une seconde base dans une pension de Cologne. Pour prix du passage illégal de ville à ville, les procès-verbaux mentionnaient des montants qui allaient de 2.000 à 10.000 francs belges ou de 650 à 2.500 R eichsmark, selon les moyens financiers des personnes à faire transiter. Le nombre

\footnotetext{
${ }^{20} \mathrm{Cfr}$. H. D. Arntz, J udenverfolgung und F luchthilfe im deutsch-belgi schen Grenzgebiet, E uskirchen 1990 ; St. K irschgens, W ege durch das $\mathrm{N}$ iemandsland. D okumentation und A nalyse der Hilfe für F lüchtlinge im deutsch-bel gi sch-niederländischen Grenzland in den J ahren 1933-1945, B onn 1998.
} 
des J uifs amenés en B elgique de cette façon, indiquait-on, était de 30 à 40 par semaine. Cependant, les fonctionnaires de la Sûreté publique de B ruxelles ne cachaient pas qu'à leur avis «la bande Sch. [était] soutenue par la police à A nvers ou que tout au moins celle-ci, tout en étant au courant, ferm[ait] les yeux ». Pour leur part, les victimes peut-on lire - «manifest[ai]ent une répugnance visible à porter ces faits à la connaissances des autorités, ne voulant pas "livrer des J uifs aux mains des non-J uifs" ». À la fin d'un copieux dossier figure, datée d'octobre 1939, la note pour nous intéressante selon laquelle «la femme $\mathrm{H}$ ausner, belle-mère de Sch. » - mise en relation avec l'entreprise d'aide à l'expatriation -, «dont l'identité est la suivante: Wolf, Lina [sic], née à W isnitz [W isznice], âgée de 61 ans, [... ] est à Anvers et réside vraisemblablement chez Sch. ${ }^{21}$.

La veuve Lea HAUSNER, née Wolf, n'était arrivée de Cologne à Anvers que depuis peu - à l'été 1939 - en compagnie de ses filles majeures et peut-être avec l'aide de son futur gendre. $E$ n fait, elle avait alors déjà passé l'âge de 65 ans. Toutes détenaient la nationalité polonaise, bien que Lea Wolf eût immigré en Allemagne longtemps avant la Première Guerre mondiale et que les enfants fussent nées à Cologne entre 1903 et 1920. Le fils unique, L asar H ausner, se trouvait manifestement en Belgique dès 1938 ; il fut déporté en 1942. L'une des filles se maria à Anvers avec le Sch. précité; tous deux échappèrent à la déportation. Sara H ausner-N eiss, la fille aînée, qui était également mariée et fut également envoyée à A uschwitz avec sa famille en 1942, ainsi que les cadettes Berta et J ohanna Hausner, avaient, fin août 1939, profité d'un geste exceptionnel de l'administration communale d'Anvers. P eut-être impressionnée par la guerre qui menaçait d'éclater, en tout cas à la demande du Comité juif anversois pour les réfugiés (A ntwerpsch K omiteit voor J oodsche V luchtelingen ${ }^{22}$ ), la police locale remit à un assez grand nombre de J uifs immigrés illégalement - qui auraient dû s'inscrire pour la mi-juillet au registre communal des étrangers pour échapper à leur refoulement vers l'Allemagne et obtenir un statut de résident légal, encore que temporaire - une attestation

\footnotetext{
21 Rapports et correspondance de la Sûreté publique des 16.5.-20.10.1939, AGR, Dossiers généraux de la Police des É trangers (2e versement), dos. 35.

${ }_{22}$ Ce comité est I'organisation qui succéda au K omiteit tot $\mathrm{V}$ erdegi ging der R echten der J oden créé en 1933.
} 
stipulant qu'ils n'avaient pu respecter ce délai à cause de la grosse affluence à I'H ôtel de Ville et avaient été régularisés entretemps ${ }^{23}$. L es trois sœurs $\mathrm{H}$ ausner obtinrent un certificat de ce type, qui pouvait à ce moment leur sauver la vie et les préservait d'une incarcération immédiate dans un camp de concentration en Allemagne. Si leur mère fut importunée par les autorités belges du fait qu'on la soupçonnait d'appartenir au groupe qui entourait le passeur Sch. ne ressort pas des archives. Comme ses enfants, elle se fit inscrire au R egistre des J uifs après l'offensive allemande. J usqu'à fin 1942, elle est officiellement signalée à A nvers avec ses deux filles B erta et J ohanna, qui gagnaient leur vie comme employées de bureau. Le 18 juin 1943, Lea WolfH ausner, à présent âgée de 70 ans, fut arrêtée avec sa fille de 26 ans J ohanna Hausner dans une cache à Anvers, assignée au camp de Malines au convoi XXI et déportée six semaines plus tard. Berta $\mathrm{H}$ ausner vécut la fin de la guerre en B elgique.

\section{Internements à Merksplas et à Marneffe}

Le gouvernement belge se décida en octobre 1938, dès avant le pogrom de novembre et l'arrivée récente d'un grand nombre de J uifs d'Allemagne, à mettre en place plusieurs camps pour réfugiés juifs. Cela se produisit à la suggestion du Comité d'Aide et d'A ssistance aux Victimes de l'Antisémitisme en Allemagne de Bruxelles, qui voulait prévenir des refoulements imminents et fit valoir toute une série d'arguments en faveur de cette solution : des raisons de coût, au vu de moyens financiers de plus en plus réduits; une préparation à l'émigration vers l'outre-Atlantique intégrant la formation professionnelle; la possibilité d'éviter ainsi des réactions antisémites de la population belge; l'enrayement de l'immigration illégale et enfin l'exemple d'autres pays ${ }^{24}$. En réalité, dans la ligne de sa politique antérieure, le gouvernement pouvait de cette manière déléguer une grande part de sa responsabilité en matière de réfugiés aux comités

23 Correspondance Stad Antwerpen, V reemdelingenbureel, avec annexes, 30.8.1939, AGR, Dossiers généraux de la Police des Étrangers (2e versement), dos. 756 ; y voir également la correspondance précédente entre le Comité des réfugiés d'Anvers, la Sûreté publique et le ministère de la J ustice.

24 Voir la correspondance du CAAVAA avec le ministre de la J ustice, 30.9.1938, AGR, Dossiers généraux de la Police des É trangers (2e versement), dos. 728. 
d'aide privés25. Avec le début de la guerre en 1939, les assignations se firent sous contrainte de l'É tat. Les camps de réfugiés de Merksplas (Merxplas), Marneffe et quelques autres centres mineurs ne ressemblaient en rien aux camps de concentration allemands; la presse nazie s'empressa, elle, d'établir la comparaison ${ }^{26}$. Le haut-commissaire aux réfugiés de la Ligue des $\mathrm{N}$ ations, Sir H erbert $\mathrm{E}$ merson, visita les camps belges à plusieurs reprises et attesta de leur caractère exemplaire ${ }^{27}$. On ne pourra non plus reprocher a posteriori à aucune des instances belges concernées de ne pas avoir prévu les suites fatales de semblable hébergement en camp. Nombre de réfugiés juifs parvinrent pourtant à se soustraire à l'internement, dont on fit dépendre le maintien du soutien financier. Pour d'autres commença à Merksplas et à Marneffe une odyssée des camps qui ne devait plus finir et qui mena à la déportation à Auschwitz.

Lorsque Walter ROSEBOOM, la bonne trentaine, célibataire, marchand de bétail de son état et agriculteur à Leer en Allemagne du Nord, parvint en Belgique, probablement en avril 1939, des centaines d'hommes juifs entrés illégalement dans le pays se trouvaient déjà au camp de réfugiés de Merksplas près d'Anvers. Roseboom demeura pourtant un an à B ruxelles avant d'être prié par le comité d'assistance local, début mai 1940, de se présenter, car il devait être transféré à Merksplas. Comme quarante autres J uifs autrichiens, tchécoslovaques et allemands, il refusa de se plier à cette mise en demeure ${ }^{2}$. La Police des Étrangers, renseignée par le Comité d'Aide, leur concéda un délai de trois jours, autrement l'arrestation menaçait. Les événements qui

\footnotetext{
${ }_{25}$ C'est l'historien belge $\mathrm{F}$ rank Caestecker qui a le premier attiré l'attention sur le fait important que la politique des réfugiés en $B$ elgique « was to a large extent subcontracted to private actors i.e. the J ewish refugee aid committees » : N ew insights in B elgian asylum policy, 1933-1939. W ork document for workshop on refugee policy, CE GES-SOMA, 21.1.2005. (N ous remercions F rank Caestecker de nous avoir transmis ce document de travail.) Cfr. aussi F. Caestecker, Ongewenste Gasten, pp. 190 sqq., 237 sqq.

${ }^{26} \mathrm{Cfr}$. A rntz, J udenverfolgung und F luchthilfe, pp. 535 sqq.

27 Voir articles de presse des 10.2.1939 et 29.4.1940, AGR, Dossiers généraux de la Police des Étrangers (2e versement), dos. 728 ; interview Emerson, Le Soir, 1.5.1940, reproduit dans B. Garfinkels, B elgique, terre d'accueil, pp. 145 sqq. ; et encore la lettre de Robert Pell, 8.3.1939, reproduite dans B eiträge zur nati onalsozi alisti schen Gesundhei ts- und Sozialpolitik, n 15, 1999, pp. 139-142 ; Pell était collaborateur du Comité d'É vian pour les réfugiés politiques de Londres (IGC).

28 «Liste des R éfugiés qui ne sont pas venus au transport pour le centre de Merxplas », $s . d$. [9.5.1940], AGR, Dossiers généraux de la Police des Étrangers (2e versement), dos. 728 ; voir aussi, ibid., la note manuscrite de la Sûreté publique, 29.4.1940.
} 
suivirent - I'invasion de la B elgique par la W ehrmacht - rendirent il est vrai caduque cette injonction. Durant les années de l'occupation, Walter R oseboom habita la commune de Schaerbeek. II se fit inscrire au « registre des J uifs » et au fichier de la communauté obligatoire (A) B), et fit prolonger pour la dernière fois en mars 1943 sa carte d'identité d'étranger, qui le désignait comme juif. II se peut qu'il ait jusque là poursuivi une existence légale. Son chemin croisa alors celui de son frère cadet I wan Ludwig ROSEBOOM ${ }^{29}$. De ce dernier, nous savons qu'en 1935, dans sa ville natale de Leer, il fut poursuivi pour «R assenschande» (relations sexuelles prohibées) et que la presse nazie I'attaqua ouvertement dans des articles antisémites provocateurs. En 1938 au plus tard, il se rendit au grand-duché de Luxembourg, où il travailla officiellement encore jusque février 1943 comme laitier et ouvrier agricole, avant, en mai de la même année, à présent à Bruxelles, de faire l'objet d'une instruction de la part du D evisenschutzkommando. Un mouchard avait fait savoir qu'l wan R oseboom devait être «en possession d'or et de devises ». Lors de la perquisition à son domicile, on ne trouva que des montants dérisoires en devises étrangères, mais une somme respectable en francs belges, dont le mouchard toucha $10 \%$ à titre de salaire, mais R oseboom fut - ainsi qu'il est dit dans les archives - " mis à la disposition du SD de Bruxelles aux fins d'acheminement à la mise au travail $\gg 30$. Comme les deux frères W alter et I wan L udwig R oseboom furent placés le même jour, le 6 mai 1943, sur la liste du X X I e convoi de Malines, on doit admettre que le DSK les appréhenda ensemble: divers indices donnent à penser qu'ils avaient vécu dans la clandestinité.

Le deuxième centre pour réfugiés d'une certaine importance se trouvait à Marneffe en Wallonie, entre Liège et Namur. Des familles juives y furent internées. Parmi elles, Chana HALPERN, épouse W eiss, et sa fille $E$ sther, qui avaient fui L eipzig début 1940 - donc pendant la guerre - avec leur époux et père J acob Weiss. Tous trois étaient de nationalité polonaise. J acob WEISS était né en 1876 à Brody, Chana Halpern en 1887 à Wysocko. À l'évidence, la famille était parvenue par une voie discrète en B elgique, car fin mars 1940 mère et fille durent

\footnotetext{
${ }^{29}$ Staatsarchiv Aurich, R ep. 16/1, Nr. 285 ; Stadtarchiv Leer, R ep. 1, A kte 3347 ; Archives nationales du L uxembourg, Police des É trangers, 248996.

30 DSK, dossier I wan R oseboom, 8.5.1943 ff., CE GE S, AA 585/111.
} 
se justifier devant un tribunal correctionnel bruxellois d'avoir enfreint les dispositions légales touchant les étrangers, renforcées en 1939 au début de la guerre, qui prévoyaient entre autres la condamnation judiciaire et l'internement forcé des immigrants illégaux ${ }^{31}$. Sans doute bientôt relaxée de Marneffe, Chana $\mathrm{H}$ alpern-W eiss vécut avec son mari un certain temps à dater de 1941 rue de la Poste, dans la commune bruxelloise de Saint-J osse-ten-Noode. De la fille, qui avait peut-être été cachée, on ne trouve pas trace.

Entre mai 1941 et juin 1943, le couple toucha une aide sociale hebdomadaire d'un montant de 100 à - finalement - 145 francs belges, comme il ressort des comptes soigneusement tenus du ménage, que l'on conserva au camp de Malines. Cette somme suffisait à vrai dire à peine à entretenir la famille. La dernière inscription qu'on y relève date du 4 juin 1943; deux jours plus tard, tous deux furent arrêtés à leur domicile légal rue de la Poste et, peu après, amenés à Malines à la caserne Dossin, où on les enregistra le 8 juin. I Is furent assignés au XX I e convoi de déportation. Sur les circonstances de leur arrestation, un témoignage fournit des renseignements: il fut donné après guerre par I sraël Dienstag, arrêté en même temps que le couple WeissHalpern.

\section{Les expulsions de mai 1940 et l'internement dans les camps du Midi de la France}

I sraël DIENSTAG, né à Bursztyn en Pologne en 1911, qui avait fui I'Autriche après l'A nschluss et était arrivé en Belgique en mai 1938, avait figuré sur la liste évoquée plus haut des bénéficiaires de l'aide du Comité juif de Bruxelles. Lui aussi appartint aux déportés du X X I e convoi, mais, transféré en 1944 au camp de concentration de Dachau, il connut la libération par les A méricains et fut rapatrié en Belgique. $L$ 'histoire mouvementée de cet homme jusqu'à son arrestation en juin 1943 commença en 1940 avec son expulsion de B elgique vers la F rance.

Le 10 mai 1940, quand la Wehrmacht envahit la Belgique, les autorités belges ordonnèrent l'arrestation et l'enfermement approxi-

\footnotetext{
31 Correspondance Sûreté publique, 22.3.1940, A GR , D ossiers généraux de la Police des É trangers (2e versement), dos. 728 ; la lettre avait trait à une ordonnance à la base de laquelle on trouve la loi belge, renforcée, sur les étrangers du 28.9.1939.
} 
mativement 10.000 "étrangers hostiles », surtout mais pas exclusivement, des réfugiés juifs d'A utriche et d'Allemagne, tous des hommes, qui en accord avec l'armée française furent expulsés vers la F rance ${ }^{32}$. Au terme d'une aventure qui les vit transiter par plusieurs camps français, les convois s'arrêtèrent finalement à Saint-Cyprien sur la côte méditerranéenne, où rien n'était prévu pour recevoir les malheureux ${ }^{33}$. É taient aussi frappés par cette expulsion des J uifs qui se trouvaient à ce moment dans l'un des camps belges d'internement ou de réfugiés34. Dans les mois qui suivirent l'éclatement de la Seconde Guerre mondiale, ils s'étaient constamment présentés au gouvernement belge comme une main-d'œuvre qualifiée35. Plus de $8.000 \mathrm{~J}$ uifs étrangers avaient suivi un appel d'organisations juives à se porter volontaires de guerre potentiels ${ }^{36}$. Ils étaient à présent soupçonnés de former une "cinquième colonne" et expulsés du territoire. Après que le camp de Saint-Cyprien dans le Midi de la France eut dû être démantelé en raison de son état catastrophique, on relégua en grande partie les internés juifs au camp de Gurs, au pied des Pyrénées. B eaucoup purent

\footnotetext{
32 Cfr. M. Steinberg, L'É toile et le fusil. T . I : L a question juive 1940-1942, B ruxelles, 1983, pp. 85 sqq. ; S. Meunier, Les J uifs de B elgique dans les camps de Sud-Ouest de la F rance, 1940-1944, mémoire de licence en histoire, ULB, B ruxelles, 1999 ; Chr. E ggers, U nerwünschte A usländer. J uden aus D eutschland und M itteleuropa in französischen I nternierungslagern 1940-1942, Berlin, 2002, pp. 65 sqq., 235 sqq., ainsi que, tout récemment, M. B ervoets-Tragholz, La liste de SaintCyprien, B ruxelles, 2006.

${ }^{33} \mathrm{~N}$ ous avons exploité le fichier, conservé, du camp de Saint-Cyprien, où sont consignés les noms de plus de 4.200 personnes, parmi lesquelles 3.400 furent enregistrées comme juifs; 1.900 d'entre eux avaient auparavant séjourné à Bruxelles, plus de 1.000 à Anvers; quelques centaines venaient des camps belges de Merksplas, Marneffe et $\mathrm{Hal}$; AD Pyrénées-Orientales, Perpignan. ( $N$ ous remercions la directrice des archives, Christine $L$ angé, de nous avoir aimablement permis la consultation, ainsi que Laurent Ducros pour diverses informations.) Parmi les réfugiés juifs internés à Saint-Cyprien et plus tard à Gurs se trouvaient aussi le peintre Felix Nussbaum d'Osnabrück et l'Autrichien $\mathrm{H}$ ans Maier (J ean Améry), qui a consigné ses mémoires dans U nmei sterlicheW anderjahre, Stuttgart, 1989, pp. 49 sqq. ; Örtlichkeiten, Stuttgart 1980, pp. 49 sqq. 34 Sur le démantèlement de ces camps en mai 1940 et l'évacuation des internés, voir à présent $R$. Van Doorslaer (É d.), La B elgique docile. Les autorités belges et la persécution des J uifs en B elgique pendant la Seconde Guerre mondiale, Rapport final CEGES, Bruxelles, 2007, chap. 5 (E. Debruyne).

35 Voir e. a. la lettre du ministère du Travail et de la Prévoyance sociale au ministre de la J ustice, 22.12.1939, ainsi que les listes correspondantes d'universitaires, d'ouvriers et de mineurs des camps de Marneffe, Merksplas et E ksaarde, 22.12.1939-29.1.1940, AGR, D ossiers généraux de la Police des É trangers (2e versement), dos. 728.

${ }^{36}$ Le F oyer israélite (Gottschalk et al.) au ministre de la J ustice, avec annexe « R elève statistique des J uifs étrangers demandant à se mettre à la disposition du Gouvernement belge», 25.9.1939, A GR, D ossiers généraux de la Police des É trangers (2e versement), dos. 785.
} 
s'en échapper, quelques-uns rentrèrent en Belgique occupée, d'autres furent enrôlés à partir du printemps 1941 dans les Groupes de Travailleurs étrangers (GTE) du régime de Vichy. En août 1942 commença l'extradition de J uifs étrangers et apatrides de la zone Sud non occupée vers la zone sous contrôle allemand et beaucoup de réfugiés expulsés de Belgique en mai 1940 se retrouvèrent dans les trains de déportés qui conduisaient de Drancy près de Paris à Auschwitz ${ }^{37}$.

I sraël Dienstag fut d'abord interné au Vigeant, un camp militaire français situé au sud de Poitiers, ensuite à Saint-Cyprien ${ }^{38}$. II doit s'en être enfui - à moins que ce ne soit plus tard -, car au printemps 1942 il était à nouveau signalé à B ruxelles, rue de la P oste à Saint-J ossetenNoode. De la mi-juillet au début d'octobre 1942, le vulcanisateur diplômé Dienstag figure sur la liste des salariés de la firme allemande J ulius Berger, qui occupait des travailleurs forcés juifs au mur de I'A tlantique dans le nord de la F rance. L'administration militaire avait créé les conditions préalables, en recrutant à partir de juin 1942 des J uifs pour ce travail extrêmement dangereux - une mesure antijuive peut-être aussi destinée à faciliter les déportations qui devaient commencer peu après. Nous ignorons de quelle manière Dienstag échappa à la déportation à l'automne 1942. En tout cas, il ne fut arrêté que le 6 juin 1943 à son adresse connue en même temps que les époux Chana Halpern-W eiss et $\mathrm{J}$ acob Weiss et amené à Malines quelques jours plus tard. Les indications sur son arrestation qu'il a fournies après guerre - il a survécu à la déportation - devant la commission belge d'indemnisation et comme témoin lors de l'instruction à l'encontre de l'ancien chef de camp Frank, divergent. Pour autant qu'il s'en souvienne, une voisine d'étage l'avait dénoncé, ce qui présupposerait que Dienstag se tenait caché dans son propre appartement. L'arrestation proprement dite aurait été le fait soit de gestapistes soit de rexistes, membres d'un groupe fasciste de collaborateurs. Dans la cave de la Gestapo à l'avenue Louise, où il resta enfermé quelques jours, il n'aurait, dit-il, été ni interrogé ni battu, et pas davantage à

\footnotetext{
${ }^{37} \mathrm{R}$ ien qu'avec les convois $n^{\circ} 24$ et 25 des 26.8 et 28.8 .1942 respectivement, ce sont au moins 120 réfugiés d'A utriche et d'Allemagne qui furent déportés à Auschwitz, dont la dernière adresse était Bruxelles ou Anvers et qui avaient été un temps internés à Saint-Cyprien ; cela ressort de I'examen du fichier du camp et des listes de déportation.

${ }^{38}$ AD Pyrénées-Orientales, Perpignan, 2619 W 1.
} 
Malines. Les Allemands se contentaient de la déportation des J uifs, dont ils savaient qu'elle signifiait la mort. Pourtant, à la question de savoir ce que l'on disait aux déportés du but du voyage, Dienstag répondit qu'il n'avait pas entendu les Allemands dire que ceux qui arrivaient à Auschwitz allaient à la mort. Au contraire, ils étaient censés aller travailler en Pologne39.

L'aide-fourreur J ames MODDE L, né à B erlin en 1904, qui avait fui à Anvers au début 1939 en compagnie de sa femme Edith HORWITZMODDEL (née en 1905) et de ses deux filles Ingeborg et Evelina, fut également expulsé de Belgique le 10 mai 1940 et interné à SaintCyprien 40 . Expédié de là au camp de Gurs, il s'échappa et, au plus tard début 1942, revint à Bruxelles, où il était officiellement signalé à Schaerbeek avec sa famille. Que Moddel se soit vu épargner d'abord la déportation à l'E st peut avoir tenu au fait qu'il avait travaillé au cours du second semestre 1942 pour une firme de B ruxelles qui fabriquait des vestes en fourrure pour la W ehrmacht. II portait sur lui un certificat en ce sens lorsqu'en avril 1943 il fut pourtant arrêté. Prévu en compagnie de sa femme et de ses enfants pour le $X X$ e convoi de déportation, il put, lui et sa fille aînée I ngeborg, 14 ans, sauter du train - comme plus de 200 hommes et femmes de ce convoi - tandis que $\mathrm{E}$ dith $\mathrm{H}$ orwitzModdel et Evelina Moddel, 10 ans, continuaient leur route vers Auschwitz. Mais le père et pas davantage la jeune Ingeborg ne devaient échapper aux nazis. Tous deux furent arrêtés à nouveau et déportés par le convoi suivant, le 31 juillet 1943.

\section{Le séjour forcé dans le Limbourg en 1941}

Lorsque Moses David FränKEL et Chaja WALD-FrÄNKEL se décidèrent à émigrer d'Allemagne et, en août 1938, de se rendre d'abord en Belgique, pour passer d'A nvers outre-Atlantique, ils avaient procédé à d'amples préparatifs. Fränkel était né en 1899 à Krosno en Pologne (qui appartenait alors à l'Autriche) et était considéré comme apatride ; Chaja Wald, qui était venue au monde à Tarnobrzeg en 1894, possédait la nationalité polonaise. Après la

\footnotetext{
39 Interrogatoire Israël Dienstag, Auditorat militaire Bruxelles, 16.3.1949, MJ DR, Dossier Dossin-F rank.

${ }^{40}$ AD Pyrénées-Orientales, Perpignan, 2619 W 1.
} 
Première Guerre mondiale, à laquelle Fränkel participa, il acheva sa formation de coupeur et chemisier dans une école professionnelle de $\mathrm{H}$ anovre. Chaja Wald apprit à repriser le linge et coudre des boutonnières. Depuis le début des années vingt, le couple dirigeait à Berlin une entreprise d'une certaine importance, qui lui appartenait, de fabrication de literie. Tous deux doivent avoir été des gens strictement religieux, qui étaient mariés selon le rite juif. Moses F ränkel était de Iongue date membre et finit par être vice-président de la communauté hassidique berlinoise $K$ nesset J isroel, au sein de laquelle se retrouvait le judaïsme orthodoxe oriental. À dater de 1938, la famille entreprit, avec I'appui du H ilfsverein der J uden in Deutschland, son émigration vers I'Australie et, au cas où elle devrait échouer, vers l'A rgentine. C'est également le $\mathrm{H}$ ilfsverein qui recommanda la famille à la bienveillance particulière du comité juif d'aide d'Anvers (A ntwerpsch K omiteit voor J oodsche Vluchtelingen). Manifestement, Chaja Wald et son mari prirent des chemins différents pour parvenir à Anvers. E lle obtint fin juillet 1938 un visa de tourisme limité, délivré par le consulat de Belgique à Berlin, et se procura des tickets de voyage et quelques francs belges. Le 21 août, elle pénétra en Belgique via la gare d'H erbesthal. Moses Fränkel, lui, franchit illégalement la frontière. Son maigre bagage d'émigré, resté en Allemagne, devait lui être envoyé plus tard. Mais, trois mois après son arrivée à A nvers, Moses Fränkel reçut de Canberra notification de ce que sa demande d'immigration en A ustralie avait été refusée. La ville portuaire flamande s'était muée en piège.

Tandis que la W ehrmacht envahissait la Belgique en mai 1940, les Fränkel partagèrent le sort d'innombrables réfugiés juifs, qui se joignirent à l'exode vers la France. Comme le couple B endheim, dont on a déjà dit I'histoire, ils se retrouvèrent, à $L$ a $P$ anne, juste en vue de la frontière française, pris dans les combats entre l'armée britannique et des troupes allemandes. Moses F ränkel fut alors blessé et, après que lui et sa femme eussent été renvoyés à Anvers, dut subir un long traitement médical. Au début de l'année 1941, les époux reçurent un ordre d'expulsion. I ls devaient quitter endéans les quelques jours leur appartement de B erchem-A nvers et se virent assigner à résidence dans 
la localité de Zelem, en province du Limbourg, où ils furent enregistrés et - comme tous les expulsés - photographiés le 1er février ${ }^{41}$.

Dans le $X X \mid$ e convoi se trouvaient plus de cent personnes qui avaient été momentanément expulsées vers le Limbourg. II s'agissait de I'une des plus anciennes mesures antijuives émanant de l'administration militaire allemande, qui touchait avant tout les réfugiés parvenus en B elgique entre 1938 et 1940 en provenance du territoire du Reich dans ses frontières d'alors. V raisemblablement en concordance avec des projets militaires de poursuite de la guerre contre I'A ngleterre, plusieurs milliers de J uifs étrangers furent transférés de la région d'Anvers vers le Limbourg, aux alentours de Genk ${ }^{42}$. On y était fort peu préparé à l'arrivée des réfugiés, qui furent soit hébergés dans des logements collectifs, soit répartis sur d'autres communes plus modestes. A ppartenant aux couches les plus pauvres de la population, ils continuèrent à émarger à l'assistance publique. De strictes conditions d'enregistrement et des réactions de rejet concertées de la part de la population locale compliquèrent de surcroît la situation des réfugiés. Au cours du printemps 1941, les autorités militaires locales autorisèrent femmes et enfants à retourner à Anvers. Plus tard dans l'année, toute la procédure d'expulsion devait être annulée, mais un certain nombre de J uifs demeurèrent dans la province du Limbourg.

L es époux F ränkel, qui avaient espéré se retrouver à A nvers pour la fête de Pessah, durent patienter au Limbourg jusqu'en août 1941. On les envoya alors à B ruxelles, où ils se présentèrent entre 1941 et début 1943 à la commune de Schaerbeek et furent également inscrits comme membres de la communauté juive obligatoire (AJ B). Leur situation matérielle à tous deux restait précaire. Moses $F$ ränkel essaya de gagner de l'argent en tant que négociant en déchets de fourrure. Son revenu mensuel tournait autour de l'équivalent de 80 Reichsmark. En mars

\footnotetext{
${ }^{41}$ Sur beaucoup de clichés, les personnes photographiées tiennent en main une ardoise d'écolier avec leur nom et leur date de naissance.

$42 \mathrm{Cfr}$. M. Steinberg, L a question juive, pp. 91 sqq. ; id., L a P ersécution des ) uifs en B elgique ( 19401945) , B ruxelles, 2004, pp. 134 sqq. ; L. Saerens, V reemdelingen in een wereldstad. E en geschiedenis van A ntwerpen en zijn joodse bevolking (1880-1944), Tielt, 2000, pp. 557 sqq., ainsi que R. Van Doorslaer (dir.), La B elgique docile, Bruxelles, 2007, chap. 8.6 (E. Debruyne). Les expulsions d'A nvers ne relevaient pas d'une décision de la F eldkommandantur locale, comme le pensent tous les auteurs, mais d'un arrêté secret du commandant militaire von Falkenhausen pris en date du 12.11.1940 dans le contexte du blocus du littoral ; Bundesarchiv-Militärarchiv F reiburg, R W $36 / 8$.
} 
1942, il fut dénoncé par un informateur du D evisenschutzkommando et I'inspecteur des douanes $\mathrm{H}$., de la branche bruxelloise du DSK, engagea une instruction à l'encontre de Fränkel pour «trafic illégal de devises et de diamants en vrac ». A uditionné après son interpellation à la gare d'Anvers, il dut expliquer la provenance des 403 Reichsmark saisis sur lui. F ränkel décrivit alors ses conditions d'existence : «C'est en août 1938 que j'ai émigré de Berlin à Anvers. L'émigration s'est faite illégalement. J e n'avais à cette occasion emporté absolument aucun argent. À Anvers, j'ai d'abord été, avec ma famille, soutenu par une organisation caritative juive. En mai 1940 [lire: janvier 1941], j'ai été évacué par les autorités d'occupation vers Zelem en province de Limbourg ; là aussi, j'ai vécu d'aides. J 'y ai séjourné jusqu'en fin août 1941. Le 1er septembre 1941, j'ai loué mon logement actuel à B ruxelles. J e gagne ma vie principalement en achetant et en vendant des fourrures et des peaux. À côté de cela, j'ai de temps en temps vendu des affaires de mon ménage. » C'est ainsi qu'il avait, expliquait-il, accepté de l'argent du R eich lors de la vente d'une montre à un soldat allemand, bien que ce fût interdit en Belgique, parce que - comme il est dit dans le procès-verbal d'interrogatoire établi par le DSK - il «ne voulait pas laisser échapper l'affaire». À Anvers, il avait voulu acheter des vivres et aussi acquérir quelques bonnets de fourrure. II ne savait pas qu'il aurait dû déclarer à la banque allemande d'émission le montant qu'il venait de percevoir. Mais cela, l'inspecteur des douanes H., qui, comme plusieurs de ses collègues, était un antisémite déclaré, ne voulut pas le lui passer. De plus, il vit dans la vente d'objets personnels sous le coup de la nécessité un comportement d'usurier juif. On lit dans son rapport final : «On doit rejeter comme invraisemblable pareille argumentation de la part d'un J uif. On sait que les J uifs sont d'habitude parfaitement instruits des dispositions en vigueur en matière de devises. Fränkel a intentionnellement passé outre à ces dispositions; selon ses propres informations, il avait réussi à placer à des conditions extrêmement favorables pour lui une montre plaquée or. [... ] L e soldat ne peut dès lors qu'avoir été lésé par ce] uif. »43

En dépit de cette instruction le concernant, Moses $F$ ränkel fut ensuite «relaxé ». La perquisition de son domicile ne donna aucun

${ }^{43}$ DSK, dossier Moses F ränkel, 17.3.1942 ff., CE GE S, AA 585/53/11. 
résultat; on ne put lui imputer un trafic de diamants. II évita sagement d'obtempérer à plusieurs mises en demeure de se présenter pour purger une peine de prison dont il avait été frappé entretemps. II laissa s'écouler jusqu'au début 1943 tous les délais qu'on lui fixait. E n mai de la même année, il fut à nouveau arrêté avec sa femme Chaja, pour être cette fois incarcéré au camp de Malines. Dans les semaines qui précédèrent le départ du X X I e convoi pour A uschwitz - les épouX furent placés sur la liste de déportation avec les numéros 100 et 101 -, ils reçurent par la Poste des dons de nombreux amis et connaissances. Après la guerre, les autorités belges attestèrent que Moses David F ränkel et Chaja Wald sont « décédées en un endroit inconnu entre le 31.7.1943 et le 1.6.1945».

\section{L'expulsion vers la F rance en 1942}

C'est un épisode fort peu connu que représentent les expulsions de Belgique vers la F rance et inversement qui commencèrent à partir de fin 1941 à l'initiative des autorités allemandes d'occupation des deux pays et en collaboration avec les services français et belges. Les responsables du processus à l'administration militaire de Bruxelles étaient les fonctionnaires du groupe «P olizei », von $\mathrm{H}$ ahn et Leiber, qui vérifiaient et approuvaient à chaque fois les listes de personnes ${ }^{44}$. Les expulsions réciproques devaient toucher des personnes avec antécédents judiciaires ou autrement indésirables et explicitement les seuls ressortissants belges ou français ${ }^{45}$. Le transfert avait lieu sous la surveillance des gardes-frontière allemands entre $\mathrm{H}$ eer-A gimont (situé au sud de Dinant) et Givet. Mais les Allemands en vinrent de plus en plus à expédier au-delà de la frontière des étrangers et, dans quelques cas, des J uifs étrangers. C'est ainsi que Sydonia SCH WARZ et son mari Hersch HERSCHDÖRFER se retrouvèrent sur la liste des personnes à expulser vers la France le 20 août 1942. L e couple, originaire de D olina et Lemberg, antérieurement de nationalité polonaise, était arrivé

\footnotetext{
${ }^{44} \mathrm{Cfr}$. I. Meinen, «L es autorités allemandes d'occupation et I'AJ B », dans J .-Ph. Schreiber et R . Van Doorslaer (É ds.), L es Curateurs du ghetto. L'A ssociation des J uifs en B elgique sous I'occupation nazie, B ruxelles, 2004, pp. 57-90, ici pp. 59 sqq.

45 Militärbefehlshaber Belgien, MVChef (von Hahn) au ministère de la J ustice, Police des Étrangers, 22.10.1941, AGR, Dossiers généraux de la Police des É trangers (2e versement), dos. 1191.
} 
illégalement de Vienne à Bruxelles au milieu de 1938, alors que tous deux avaient déjà un âge avancé, en même temps que trois fils adultes et leurs familles. Durant l'exode au printemps 1940 ou plus tard, les parents s'enfuirent en $\mathrm{F}$ rance avec leur fils Otto, mais étaient rentrés de Paris à Bruxelles pour le début 1942. C'est là que Hersch $\mathrm{H}$ erschdörfer, confiseur diplômé, et sa femme s'installèrent rue des Tanneurs - une rue habitée principalement par des réfugiés juifs et située dans le quartier populaire des Marolles, au centre-ville - à proximité immédiate de leurs enfants. Peu après, les familles se retrouvèrent logées ensemble à l'étroit dans les M arolles; $\mathrm{H}$ erschdörfer était gravement malade. Fin juillet 1942 - à ce moment se préparait le premier convoi de Belgique vers Auschwitz - lui et Sydonia SchwarzHerschdörfer devaient avoir été informés qu'on voulait les expulser. Les archives ne fournissent pas les raisons de cette mesure: elles pourraient avoir été en rapport avec leur séjour temporaire en $F$ rance. Toujours est-il que le mari, qui souffrait manifestement de tuberculose pulmonaire, se fit établir à plusieurs reprises et en langue allemande un certificat médical attestant qu'il était placé sous surveillance et devait éviter tout effort ${ }^{46}$. Mais, le 19 août, la gendarmerie de Bruxelles reçut instruction d'interpeller les conjoints, de les amener le lendemain à la frontière à $\mathrm{H}$ eer-A gimont et de les y remettre aux gardes-frontière aux fins d'expulsion vers la $\mathrm{France}^{47}$. Des documents de la gendarmerie nationale et une lettre de la Police des Étrangers belge à I'administration militaire établissent la remise en date du 20 août 1942 des deux personnes - et d'autres - aux gardes-frontière allemands. Tous deux doivent pourtant avoir regagné la B elgique, à moins que $H$ ersch Herschdörfer ne soit décédé lors de l'expulsion. Sydonia Schwarz, veuve, 52 ans, fut appréhendée à nouveau à B ruxelles en mai 1943 et transférée à Malines. Elle a survécu à la déportation du 31 juillet 1943. Son fils aîné Arthur, la femme de ce dernier R uchla Szpic$\mathrm{H}$ erschdörfer et leur enfant, L eonard $\mathrm{H}$ erschdörfer, venu au monde à B ruxelles en mars 1943 et âgé de 4 mois seulement, qui avaient été arrêtés en même temps qu'elle et se trouvaient dans le même convoi,

\footnotetext{
${ }^{46} \mathrm{~L}$ a carte d'enregistrement AJ B du couple Herschdörfer-Schwarz porte également le timbre «F » (freigestellt), c'est-à-dire que ni I'un ni l'autre ne devait pour l'heure être déporté.

47 L ettre du ministère de la J ustice, Police des É trangers à la B rigade de Gendarmerie B ruxelles, 19.8.1942, A GR, D ossiers généraux de la Police des É trangers (2e versement), dos. 1191.
} 
furent assassinés. Les deux fils cadets, Otto et Samuel H erschdörfer, avaient été déportés dès septembre 1942 et eux aussi périrent à Auschwitz.

\section{Aide à la fuite de B elgique en F rance, 1941-1942}

I cek GOLDBE R G, veuf, né en 1894 à Piotrków (Pologne) et qui avait émigré en 1920 vers la B elgique, était libraire et relieur à Anvers, où grandirent également ses enfants Max, $H$ élène et $R$ uben. À partir de 1941, il se consacra à la fabrication de valises munies de compartiments secrets ou de doubles fonds qui pouvaient être utiles à des réfugiés. C'est une valise de ce genre qu'il vendit à Walter RUBENS, un drapier d'Aix-la-Chapelle, qui avait quitté l'Allemagne dès 1933. $R$ ubens voulait tenter, en compagnie de sa femme Berta LINDHEI MER, de sa mère et de sa bellemère, de passer de Belgique en $\mathrm{F}$ rance non occupée, et de là en Suisse. Le fils aîné de Goldberg, Max Goldberg, un cliveur de diamant de 20 ans, devait à cette occasion assumer la tâche de passeur et de « guide » - il n'en était pas à son coup d'essai puisqu'il avait, en 1941 déjà, conduit des réfugiés dans le Midi de la F rance. E $n$ juin 1942, le groupe, qui avait été trahi par un mouchard, fut arrêté en gare du Nord (Bruxelles) à bord d'un train à destination de Courtrai par des fonctionnaires du Devisenschutzkommando et remis à la Sicherheitspolizei. Chacun des intéressés détenait des faux passeports. Lors de l'inspection des bagages, on trouva des objets de valeur et des devises. Walter $\mathrm{R}$ ubens en fut reconnu comme le propriétaire.

Auditionné par le DSK, Rubens déclara: " $\mathrm{En}$ faisant connaissance avec Max Goldberg I'an dernier [1941], j'ai aussi fait connaissance avec son père, qui habitait alors à Anvers, Lange $\mathrm{K}$ ievitstraat. J 'avais entendu dire qu'il fabriquait des valises à poches secrètes. Comme je détenais déjà avant guerre de nombreuses pièces d'or et que je considérais les poches secrètes de ces valises comme les plus sûres cachettes pour ces pièces, je me suis également fait fabriquer à l'époque par Goldberg une valise à compartiment secret. J 'ai emporté moimême chez Goldberg les pièces d'or en ma possession ; en ma présence, il a introduit les pièces dans le montant en bois d'une valise. Depuis lors, les pièces se trouvent dans cette valise. Dans la perspective de mon émigration, cette valise était vraiment ce qu'il fallait pour 
emporter ces pièces d'or. [... ] J e voulais emporter ces pièces d'or avec moi en Suisse et y assurer grâce à elles de quoi vivre. »48

L orsque le tribunal de la F eldkommandantur d'A nvers condamna Walter Rubens fin août 1942 et confisqua ses objets de valeurs «par décision administrative, au profit de l'administration militaire allemande », c'est-à-dire tout bonnement les vola, lui-même, sa mère et sa belle-mère avaient déjà été emmenés à Auschwitz - par le l er convoi, qui partit de Belgique le 4 août 1942. Comme il fut soutenu par le Dr. S., conseiller près le tribunal militaire, qui mena le macabre procès, l'accusé ne pouvait être présenté à l'audience au fond, «parce qu'il avait été envoyé fin juillet à l'Est sans destination connue par le Sicherheitsdienst aux fins de mise au travail $\gg 49$. Le passeur, Max Goldberg, fut également déporté; il prit le chemin d'Auschwitz à la mi-septembre par le convoi IX . Contre son père, le fabricant de valises à compartiments secrets, une instruction pour «contrebande de devises et d'êtres humains », qui avait déjà débuté fin 1941, était à ce moment pendante.

Dès ce moment, le DSK d'A nvers avait mis plusieurs informateurs sur la piste des J uifs qui tentaient de fuir vers la F rance. C'est de cette manière que les enquêteurs de la douane avaient eu vent de ce qu'un « convoi de J uifs », comme l'appelait le rapport du DSK, devait avoir lieu au départ de la gare de Bruxelles-Midi. En fait, un groupe d'émigrants illégaux fut arrêté début novembre 1941 avec un complice belge suite à ces informations, et I cek Goldberg se retrouva aux mains des Allemands: "Lors de la perquisition de l'appartement et de I'atelier de l'accusé Goldberg, on mit la main sur trois valises à poches secrètes terminées et deux à demi terminées. C'est Goldberg lui-même qui les avait fabriquées. Selon ses dires, il voulait vendre les valises à des gens qui avaient l'intention d'émigrer et, à l'aide des valises, voulaient transporter sans autorisation à l'étranger de l'argent et d'autres obj ets de valeurs. » 50 L 'enquête contre Goldberg entre autres, dans laquelle étaient impliqués plusieurs passeurs et faussaires et qui fut un moment menée également par la Sicherheitspolizei, ne devait

\footnotetext{
48 I nterrogatoire Rubens, 24.6.1942, dans DSK, dossier I cek Goldberg, 30.12.1941 ff., CE GES, AA 585/41/4.

49 DSK, dossier Walter R ubens, 23.6.1942 ff., CE GE S, AA 585/42/2.

50 DSK, dossier I cek Goldberg, 30.12.1941 ff., CE GE S, AA 585/41/4.
} 
mener qu'en décembre 1942 à un jugement du tribunal militaire allemand d'Anvers ${ }^{51}$. Goldberg, qui avait été bientôt relaxé de sa garde-à-vue moyennant paiement d'une caution élevée, déménagea début 1942 d'Anvers à Bruxelles. Manifestement, il avait pu poursuivre entretemps son activité d'aide à l'émigration avec l'appui de son fils Max jusqu'à l'arrestation de celui-ci en juin 1942. II fut alors condamné pour délit financier à payer une amende qui correspondait au montant de la caution. Six valises trafiquées prêtes ou à moitié prêtes qui se trouvaient en sa possession furent confisquées. Nous ne pouvons savoir s'il fut ensuite à nouveau arrêté ou livré à la Gestapo, qui poursuivit la procédure pour «trafic d'êtres humains ». Le 9 juin 1943, I cek Goldberg fut placé à Malines sur la liste du X X I e convoi, en même temps que sa fille Hélène Goldberg, 16 ans, et son fils cadet R uben (R ené), 10 ans. Le 31 juillet 1941, ils partirent pour A uschwitz et pour la mort.

Dans le contexte de l'enquête menée à charge de Goldberg, deux coïnculpés fournirent des informations sur l'organisation de l'aide rémunérée à la fuite et sur des itinéraires menant dans le Midi de la France, qu'il faut rapporter ici par extraits, encore qu'il convienne de rester prudent face à ces procès-verbaux d'interrogatoire établis sous la pression. L'entreprise, une parmi bien d'autres, semble s'être mise en branle fin 1940. On recruta à Anvers des gens qui voulaient être amenés illégalement en France non occupée et on leur fournit de fausses cartes d'identité françaises. Plusieurs passeurs («Schlepper»), juifs et non juifs, parmi lesquels Goldberg junior, conduisirent les fugitifs de B ruxelles au-delà de la ligne de démarcation qui séparait le territoire français occupé par les Allemands de la zone Sud non occupée52. Le groupe qui entourait Goldberg recourut pour ce faire à un itinéraire passant principalement par Salins (au sud de Besançon): «Par une connaissance de R. du nom de M. qui, avec le J uif [Max] Goldberg, a amené illégalement des gens dans le Midi de la France, $R$. savait que l'itinéraire qu'il empruntait via la Somme avait été

\footnotetext{
51 Voir DSK, dossier J uda L., 11.11.1941 ff., CE GES, AA 585/41/1 ; et DSK, dossier Mois R. et al., 19.1.1942 ff. , AA 585/53/9.

$52 \mathrm{Cfr}$. à ce propos E. Alary, «Les J uifs et la ligne de démarcation », dans Cahiers de la Shoah, n 5, 2001, pp. 13-49; id., La Ligne de démarcation, 1940-1944, Paris, 2003, pp. 182 sqq. ; A. Meyer, Täter im V erhör. Die "E ndlösung der J udenfrage" in F rankreich 1940-1944, Darmstadt, 2005, pp. 164 sqq.
} 
neutralisé. Lors de notre séjour à Salins, nous nous sommes rendu compte qu'il était fort simple de faire franchir illégalement à des gens la ligne de démarcation. N ous avons donc eu l'idée de faire à l'avenir passer les convois par Salins. Aux environs de fin septembre - début octobre [1941], nous sommes allés, R . et moi, accompagnés de Goldberg et de M., à Salins pour leur montrer le chemin. [... ] U ne douzaine de personnes participèrent au voyage, des gens qui voulaient être amenés illégalement au-delà de la ligne de démarcation et payaient pour ça. II ne s'agissait que de J uifs originaires d'A nvers. Trois des J uifs étaient en possession de valises qui recélaient de l'argent caché. Ces valises avaient été fabriquées par le père de Max Goldberg. » 53

Pour faire face aux débours et à titre de rémunération ( Schlepperlöhne » : telle était l'expression usuelle au DSK ), on exigea des réfugiés juifs des montants s'élevant de 4.000 à 12.000 francs belges par personne - plusieurs fois le revenu mensuel moyen d'un travailleur belge, mais en 1941 un prix tout-à-fait courant ; les tarifs continuèrent à grimper par la suite. Ceux qui, à leurs risques et périls, accompagnaient les J uifs dans leur fuite, ne conservaient le plus souvent qu'une fraction de l'argent. S'ils étaient eux-mêmes juifs, ils profitaient de l'occasion pour se mettre en sûreté dans le Midi de la France. La famille Goldberg n'y réussit pas.

\section{F uites et tentatives de fuite vers la F rance en 1942 Les déportations de Drancy}

En juillet 1942, la déportation de masse de J uifs de F rance et des Pays-Bas avait commencé et, début août, le premier convoi fut constitué à Malines. La fuite à travers plusieurs frontières et lignes de démarcation vers le Midi de la France - et de là éventuellement vers la Suisse ou l'E spagne - ouvrit à des J uifs qui étaient soumis à la domination allemande en Europe occidentale l'une des quelques possibilités de se sauver qui subsistaient.

Début août 1942, des fonctionnaires de l'antenne de Lille du $D$ evisenschutzkommando en F rance arrêtèrent dans le train de Lille à Nancy-Besançon deux J uives, Alta AltmAN épouse Ejgier et sa fille

53 Sipo-SD Brüssel, interrogatoire J ean R., 8.12.1941, dans DSK, dossier I cek Goldberg, 30.12.1941 ff., CE GE S, AA 585/41/4. 
Sura EJ GIER, ainsi que le ressortissant français $R$ ené $R$. L'interpellation était due à un renseignement fourni par un informateur, selon lequel un passeur allait faire franchir illégalement à deux J uives de Belgique la ligne de démarcation qui partageait la $\mathrm{F}$ rance pour les amener en zone libre. L'interrogatoire des deux femmes, qui se trouvaient soumises à une pression massive, amena les douaniers sur la trace d'une seconde passeuse impliquée, une ressortissante belge de Bruxelles, du nom d'I rène $M$. Informé depuis Lille, le DSK de $B$ ruxelles ouvrit une information contre elle. I rène $M$. déclara lors de son interrogatoire qu'elle n'avait fait la connaissance d'A Ita A Itman et de Sura E jgier que peu auparavant : « Les J uives voulaient émigrer en F rance non occupée. E lles voulaient me donner une rémunération si je les aidais à franchir la ligne de démarcation. J 'ai été d'accord et je leur ai promis de les amener à Lille chez un couple de mes connaissances, les D., d'où elles seraient acheminées plus loin. [... ] Le 30 juillet 1942, j'ai pris le train pour Lille avec les deux J uives. À cette occasion, j'ai emmené de Belgique en $\mathrm{F}$ rance des sommes d'argent relativement importantes. Ces sommes que j'avais emportées, je les ai remises aux J uives à Lille. [... ] À part ça, je n'ai rien à voir avec cette affaire. II est exact que j'ai aussi porté en $\mathrm{F}$ rance les deux cartes d'identité juives. [... ] J e savais qu'il est interdit d'aider des ] uifs à émigrer illégalement et qu'on ne peut emporter de l'argent de Belgique qu'avec une autorisation. Mais je voulais simplement en faisant cela gagner quelque chose pour vivre. $\gg 54$

I rène $M$. ne toucha en fait pas le salaire d'un montant de 20.000 francs belges qu'on lui avait promis. II avait été mis en dépôt et ne lui serait remis en mains propres qu'après l'arrivée des deux femmes en France non occupée. Un tribunal militaire allemand la condamna à quatre mois de prison pour délit financier et complicitéavec un délinquant cherchant à quitter sans autorisation le territoire belge.

Qui étaient ces deux femmes, qui se risquèrent à fuir au dernier moment ? Alta Rosa Altman était née en Pologne en 1898. Elle se maria dans une grande famille d'Opatów, où naquirent aussi ses deux filles Sura-R ywka et Mindla, respectivement en 1922 et 1923. En 1929, avec son mari Uszer Ejgier et les enfants, elle émigra de Lódz en

${ }^{54}$ DSK, dossier I rene M., 15.9.1942 ff., CE GE S, AA 585/54/7. 
B elgique. Les deux parents exerçaient le métier de tailleurs. Durant la période de l'occupation allemande, ils habitèrent le quartier bruxellois des Marolles, au voisinage immédiat d'une autre famille de tailleurs de six personnes du même nom, qui était également venue d'Opatów et de Lódz. U szer E jgier et sa fille cadette Mindla décidèrent dès le premier semestre 1942 de fuir en $F$ rance. IIs arrivèrent en territoire non occupé et atteignirent probablement Nices5. Fin juillet, alors que la déportation menaçait en B el gique, la mère et la fille aînée essayèrent de les $y$ suivre. Elles s'étaient procuré à B ruxelles pour 4.000 ou 6.000 francs belges de fausses cartes d'identité. Elles ne portaient pas I'«étoile juive». Avec l'appui de la passeuse I rène M., elles avaient réussi à quitter la Belgique et, pendant le trajet en train de Lille, elles avaient - si I'on en croit la plainte du DSK - caché leur argent «de manière extrêmement sophistiquée ». Les fonctionnaires allemands trouvèrent également les bons documents portant le timbre « J uif - J ood » cachés dans les bagages - ils avaient été encollés dans une couverture de livre. Un mois environ après l'arrestation, en septembre, le DSK de Lille informa la Sicherhei tspolizei de la tentative de fuite et requit en même temps auprès du tribunal de la $\mathrm{Feldkommandantur} \mathrm{locale} \mathrm{de}$ condamner les deux femmes à une peine de prison de huit mois chacune. Toutes deux furent amenées pour purger leur peine à la prison de Saint-Gilles à Bruxelles et, le 20 novembre 1942 - avec I'assentiment du tribunal de la Wehrmacht de Lille, qui se fit I'auxiliaire des desseins de la Gestapo -, transférées au camp de Malines. Manifestement, elles devaient être déportées par le convoi X VIII du 15 janvier 1943. Mais elles parvinrent à obtenir un «numéro W », destiné aux détenues mises au travail. Elles furent vraisemblablement occupées un temps à l'atelier de couture du camp. Ce n'est que très tardivement, à savoir le 27 juillet 1943, que les deux femmes furent placées sur la liste du XX I c convoi de déportation. Dans la série des numéros de déportation, les noms d'Alta Altman-Ejgier et de Sura Ejgier figurent immédiatement derrière ceux de toute la famille de leurs voisins des Marolles (J udka Ejger et R ywka Grosman-Ejger avec leurs quatre enfants adultes Alter, Szmul, J osef et Sara), qui furent

55 L eurs noms ne figurent pas sur les listes de déportation de D rancy. 
également déportés à Auschwitz. Mais la question continue à se poser de savoir s'il existait un lien entre les arrestations de tous ces parents ${ }^{56}$.

La famille du fripier W olf TODER était arrivée à Anvers en 1927 en provenance de R ymanów en Pologne, d'où son épouse R egina MAN et deux de ses enfants étaient originaires. H ersch Chaïm Toder, son fils aîné, né en 1911, y apprit le métier de courtier en diamants. Sous l'occupation allemande, il gagna de l'argent comme pelletier. En avril 1942 encore, toute la famille était enregistrée dans la ville portuaire flamande. Peu après, au plus tard après le début des déportations de B elgique en août, W olf Toder, déjà âgé de 57 ans, et sa femme R egina, de cinq ans plus jeune que lui, doivent avoir préparé, avec des parents, leur fuite en $\mathrm{F}$ rance non occupée. II est établi qu'ils réussirent à sortir illégalement de Belgique et qu'ils parvinrent jusqu'à la petite ville française de Vierzon, dans le département du Cher, située immédiatement sur la ligne de démarcation ${ }^{57}$. Le couple fut arrêté probablement lors de sa tentative de la franchir clandestinement - et amené au camp d'internement de Pithiviers, situé en territoire occupé et où se trouvaient des milliers de femmes et d'enfants juifs arrêtés lors de la grande rafle opérée à Paris en juillet 1942. Le 16 septembre, les Allemands firent amener un groupe relativement important d'internés à Drancy près de $P$ aris, parmi lesquels sans doute aussi W olf Toder et R egina Man-Toder, car le lendemain on plaçait les deux sur la liste du convoi $n^{\circ} 34$, qui quitta le 18 septembre 1942 la gare du BourgetD rancy ${ }^{58}$. La majeure partie des déportés furent gazés dès leur arrivée à A uschwitz.

\footnotetext{
56 La graphie des noms de famille Ejgier (Ejger) diverge dans les documents, mais la relation de parenté nous paraît assurée.

57 C'est là qu'était installé un bureau de la Sipo-SD d'Orléans, qui faisait savoir le 30.7.1942 " qu'au cours des huit derniers jours a eu lieu une fuite massive de J uifs de $\mathrm{H}$ ollande, de B elgique et de Paris en direction du territoire français non occupé. L es J uifs étaient porteurs presque sans exception de cartes d'identité nouvellement établies, sur lesquelles ils ne sont pas mentionnés comme J uifs. En trois jours, quelque $70 \mathrm{~J}$ uifs ont par exemple été appréhendés à la gare de Vierzon par notre bureau local. On ne peut, faute de personnel, contrôler d'ici les autres gares frontalières. Mais il y a ici des informations de la F eldgendarmerie et des bureaux de la douane comme quoi des milliers de J uifs se rendent illégalement en territoire non occupé. De nombreuses officines à $\mathrm{L}$ a $\mathrm{H}$ aye, B ruxelles et $\mathrm{P}$ aris jouent les passeurs et $\mathrm{S}^{\prime}$ occupent de leur procurer de faux papiers. », Commando de Vierzon au commandant de la Sicherheitspolizei de Paris, 30.7.1942, CD J C, XXVI-51.

58 Comme lieu de résidence de R egina Man-Toder, on indiqua «Vierzon », tandis que pour son mari, c'est Anvers qui est indiqué; CDJ C (www.memorialdelashoah.org), Mms-1012244_B.jpg ;
} 
Pourquoi les fils adultes de la famille ne s'étaient-ils pas joints à la tentative désespérée de fuite, dont ils ne pouvaient connaître l'issue? La réponse semble être qu'ils se trouvaient déjà pris dans la machine de persécution. À partir de l'été 1942, H ersch Chaïm Toder - en même temps qu'un de ses frères - fut enrôlé pour le travail obligatoire à Dannes (dans le Nord de la France) au sein de l'entreprise J ulius $B$ erger mentionnée plus haut. Cette mise au travail n'était souvent que le prélude à une déportation ultérieure. Comme de nombreux autres jeunes gens affectés aux chantiers du Mur de l'A tlantique, tous deux devaient être amenés de Malines à Auschwitz par le XVI e convoi fin octobre 1942. Hersch Toder put s'enfuir mais, à la mi-décembre, il fut à nouveau arrêté à Anvers par la $\mathrm{F}$ eldgendarmerie lors d'un contrôle de rue, enfermé quelques semaines à la maison d'arrêt militaire locale et, fin janvier 1943, livré à la Sicherheitspolizei, qui le fit amener à Malines. Prévu pour le convoi de déportation suivant, le XX e, H ersch Toder fut finalement assigné au X X I e et envoyé à la mort trois mois et demi plus tard, après que les listes de déportation se fussent remplies de plus de 1.500 noms de victimes d'arrestations. II survécut sans doute d'un an à peine à ses parents assassinés à A uschwitz.

Les exemples ne sont pas rares de parents de J uifs que l'on déporta plus tard de B elgique qui avaient essayé de fuir en 1942 encore vers la F rance non occupée mais qui furent arrêtés en chemin par des agents de la police ou des gardes-frontière allemands ou français et dirigés via D rancy vers les centres d'extermination de l'E st. I nversement, nous ne savons vraiment pas combien de ces fuites devant la déportation se déroulèrent avec succès. L'histoire du XXIe convoi présente des destins individuels qui permettent de connaître tant les stratégies de sauvetage individuelles des J uifs que le caractère inexorablement étanche de l'appareil allemand de persécution.

Les frères David et Eduard STEINLAUF, nés à Trèves en 1906 et 1909, de nationalité polonaise et résidant à Anvers avec leurs parents comme tailleurs de diamants dès la fin des années 20, allèrent à Auschwitz respectivement par le convoi $n^{\circ} 7$ du 19 juillet 1942 en partance de Drancy et par le XXIe convoi en partance de Malines. David, le frère aîné, parvint à se sauver jusqu'à la région de B ordeaux,

cfr. S. K larsfeld, Le Calendrier de la persécution des J uifs en F rance 1940-1944, Paris, 2001, p. 1120. 
avant d'être appréhendé sur la ligne de démarcation ou d'être pris dans les rafles qui y eurent lieu à l'été 1942 et, le 18 juillet, sur ordre de la Sicherheitspolizei de Bordeaux, expédié à Drancy en même temps que 170 autres hommes et femmes, au nombre desquels au moins 17 réfugiés juifs de B elgique 59 . V ingt-quatre heures plus tard, le 19 juillet, les J uifs de Bordeaux partirent de Drancy pour Auschwitz par le convoi dans lequel se trouvaient aussi les premières victimes de la grande rafle des 16-17 juillet à Paris. Eduard Steinlauf, le cadet des deux frères, était resté en Belgique; il avait épousé en 1942 Mala CHAJT 31 ans, née à Opatów, et fut déporté le 31 juillet 1943 de Malines avec sa femme.

II se trouvait pourtant des J uifs de B elgique pour parvenir dans le Midi de la France. Des fuites réussies de ce genre sont également documentées dans le contexte du X X I e convoi. Parmi les déportés du 31 juillet 1943 se trouvait Szyja HeRMAN, de Varsovie, 50 ans. Travaillant dans le cartonnage, il avait vécu avec sa famille à A nvers et ensuite dans la commune de Saint-Gilles à B ruxelles. Son fils J uda Herman était né à Varsovie en 1920, sa fille Genia Herman cinq ans plus tard à Anvers. Les deux enfants, qui exerçaient le même métier que leur père, étaient encore signalés au printemps 1942 à B ruxelles et le Service des Affaires juives à l'avenue Louise avait déjà établi une fiche pour Genia H erman en vue de préparer son « expulsion ». Mais à la mi-août 1942, elle fut internée au camp de R ivesaltes, dans le Midi de la F rance, devenu entretemps camp de déportation, où la police française faisait enfermer aussi des personnes ayant franchi illégalement la frontière ${ }^{60}$. Manifestement, elle avait, comme son frère J uda, réussi à fuir dans le $\mathrm{Midi}$, alors que les trains de déportation partaient déjà de Belgique. Comme il ressort du fichier des internés de R ivesaltes, Genia H erman, 17 ans à l'époque, ne demeura qu'un mois au camp. E lle fut prétendument relaxée. P eut-être faisait-elle partie de ces enfants et adolescents qui furent sauvés de R ivesaltes par des organisations d'aide et furent hébergés dans des homes. Nous ne connaissons pas la suite de son parcours mais, à ce qu'il semble, elle échappa à la persécution. Son frère, par contre, avait été placé au

59 CDJ C (www.memorialdelashoah.org), Mms-1013904_B.jpg ; cfr. S. Klarsfeld, ibid., pp. 533, 540.

${ }^{60}$ AD Pyrénées-Orientales, P erpignan, 1260 W 14. 
camp des Milles, près de Marseille. De là, les autorités de Vichy le livrèrent début septembre en zone nord occupée et, le 9 septembre 1942, il fut déporté de Drancy par le convoi n³0.

\section{F uite des Pays-Bas}

Dans le XXIe convoi se trouvaient 101 J uifs qui n'étaient enregistrés dans aucune commune belge, mais avaient leur domicile aux Pays-Bas - pour plus des deux tiers à Amsterdam. Ces réfugiés, qui furent portés entre mai et juillet, seuls ou en petits groupes, sur les listes de déportation, doivent quasiment sans exception n'être arrivés à destination qu'en 1943 et uniquement en vue de transiter par la Belgique. Arrivés de Hollande, où la plupart vivaient depuis leur naissance, ils essayèrent d'atteindre le territoire français pour parvenir de là en Suisse ou en E spagne, lorsqu'ils furent arrêtés dans ce pays de transit que constitue la B elgique en $E$ urope occidentale.

Le 20 mai 1943, un groupe de cinq J uifs d'A msterdam se retrouva la proie de l'occupant allemand à la gare du Nord à B ruxelles: Flora COHEN, née à Amsterdam, couturière de son état, approchait des 27 ans. Dès 1941, les Allemands avaient déporté son mari des Pays-Bas au camp de concentration de Mauthausen. Liesel SONDHEIMER, 28 ans, fourreuse, était également veuve. E lle provenait originellement de la $\mathrm{H}$ esse en Allemagne et avait mis au monde en 1936 en $\mathrm{H}$ ollande son fils Wolfgang WIJ NMAN, qui fut arrêté en même temps que sa mère. Son père avait été assassiné à A uschwitz fin 1942. L ea B ATIST et L evie DUPONT étaient tous les deux nés en 1920 - elle à Berlin, lui à A msterdam - et s'étaient mariés à Amsterdam à la fin des années trente. Tous deux étaient, comme F lora Cohen, détenteurs de la nationalité néerlandaise et portaient sur eux lors de leur fuite leurs documents d'identité néerlandais, estampillés d'un grand «J». Ce n'étaient pourtant pas les vrais papiers qui scellèrent leur sort. Car leur arrestation n'eut pas lieu dans le cadre d'un contrôle d'identité. Le D evisenschutzkommando apprit par l'un de ses informateurs leur arrivée à Bruxelles, suite à quoi le secrétaire aux douanes $B$. se rendit incontinent à la gare du N ord et appréhenda les réfugiés hollandais.

L e couple B atist-D upont au moins appartenait au vaste groupe des J uifs néerlandais qui, l'année précédente encore, avaient pu espérer 
être à l'abri d'une déportation en Allemagne. En juillet 1942, lorsque les premiers trains partirent de Westerbork aux Pays-Bas pour Auschwitz, Levie Dupont travaillait comme fourreur pour l'une des entreprises qui produisaient pour la W ehrmacht. À partir d'août 1942 au plus tard, Lea Batist-D upont, qui avait appris le même métier que son mari, travaillait également comme pelletière pour l'inspection allemande de l'équipement. Fin novembre 1942 encore, elle put obtenir une nouvelle attestation établissant qu'elle était «exemptée » de la déportation. Mais à partir du début de l'année 1943, les Allemands abandonnèrent largement aux Pays-B as le système des « exemptions » massives, dont dépendait un tiers environ des J uifs néerlandais. IIs procédèrent en même temps à de grandes rafles à A msterdam et en province et accélérèrent de façon dramatique l'arrestation d'hommes, de femmes et d'enfants juifs ${ }^{6}$. D ans cette situation, les époux BatistDupont et beaucoup d'autres J uifs vivant en $\mathrm{Hollande}$ prirent au printemps 1943 le risque de fuir vers la $\mathrm{F}$ rance.

A près leur arrestation à la gare du Nord à Bruxelles, le Devisenschutzkommando livra les quatre adultes et l'enfant de 6 ans à la Sicherhei tspolizei. À peine avaient-ils été amenés à Malines que les A llemands arrêtaient la mère veuve de L evie Dupont, Duifje ZEGERIUS, et ses frère et sœur cadets J udith et J opie, respectivement 18 et 13 ans. Eux aussi avaient fui Amsterdam, eux aussi tombèrent en Belgique aux mains de leurs persécuteurs. Aucun d'entre eux ne faisait partie des survivants du X X I e convoi.

Que plusieurs proches d'une famille néerlandaise qui tentait de fuir vers la F rance tombent I'un après l'autre en B elgique au pouvoir de la Gestapo n'a rien d'un cas unique. Rebecca KESING-DE VOS, née à A msterdam en 1907 et femme au foyer, s'enfuit vers la B elgique avec son mari, de deux ans son aîné, et leurs trois petits enfants, en vue de gagner la Suisse via la F rance. On ne peut préciser quand ils quittèrent la $\mathrm{H}$ ollande, mais la famille résida à coup sûr un certain temps illégalement en B elgique et y attendit un moment favorable à la poursuite de son voyage. Durant I'hiver 1942-1943, la mère, sa fille de 8

\footnotetext{
61 Un tiers environ du total des 107.000 J uifs déportés des Pays-B as partit pour Sobibór entre mars et juillet 1943. Pour une vue synthétique, cfr. P. Griffioen - R. Zeller, «L a persécution des $J$ uifs en Belgique et aux Pays-Bas pendant la Seconde Guerre mondiale. Une analyse comparative. », dans Cahiers d'H istoi re du T emps présent, $n^{\circ}$ 5, 1999, pp. 73-132.
} 
ans, R oosje Y vonne, Ingrid Marietta, 7 ans, et K ees, 6 ans, furent arrêtés dans leur cachette, amenés sans doute au début de février 1943 à Malines et enregistrés pour le $X X$ e convoi. Le père de famille put échapper à l'arrestation. Goedman K ESING avait des relations dans le pays, qu'il avait visité avant-guerre comme voyageur de commerce, et trouva de l'appui auprès d'un ancien partenaire commercial belge à E tterbeek (B ruxelles) et de sa femme. Tandis qu'il devait se cacher luimême, K esing, qui s'était procuré de faux papiers d'identité belges, entreprit entre février et avril 1943 des efforts désespérés pour faire sortir sa femme et ses enfants de Malines. Un contact avec une femme habitant Bruxelles, qui prétendait pouvoir corrompre un officier SS, promit une chance de salut. La femme exigea beaucoup d'argent, que $K$ esing paya, non sans prendre diverses mesures de précaution. Mais il se fit escroquer. Les délais se multipliaient, au terme desquels la libération était censée avoir lieu. Le 19 avril, le X X e convoi partit pour A uschwitz avec à son bord R ebecca K esing-De V os et ses trois enfants. $K$ esing reçut par contre l'information que sa famille avait été mise en sûreté à un troisième endroit. Bien qu'il redoutât depuis un certain temps d'être trahi, il continua à espérer plusieurs jours la nouvelle de la libération des siens, alors que ceux-ci avaient déjà été assassinés par les Allemands. Au début mai 1943, il décida de porter plainte pour escroquerie auprès de la police belge. II rédigea un compte rendu exhaustif des événements et des tractations qu'il avait menées avec cette femme entre février et mai, et déposa le document chez un avocat de B ruxelles. Vers la fin juin 1943, Goedman K esing fut arrêté alors qu'il voulait regagner sa cachette. Un mois plus tard, les Allemands le déportèrent à A uschwitz par le X X I e convoi.

\section{Conclusion}

B eaucoup de victimes du $X X \mid$ e convoi se trouvaient en fuite depuis 1938-1939, lorsque la violence déchaînée par les nazis les avait chassés d'A utriche, de Tchécosl ovaquie ou d'A llemagne. Les tentatives de fuir s'étendent jusqu'à l'époque des déportations entre 1942 et 1943. À ce moment, les Allemands faisaient le maximum pour empêcher que les J uifs s'échappent hors de la zone qu'ils contrôlaient et les noms des réfugiés interceptés sur la route qui menait de B elgique ou des Pays- 
Bas en F rance se retrouvèrent sur les listes de déportation dressées à Malines. Comme bon nombre de J uifs tombèrent aux mains de la Sicherheitspolizei allemande alors même qu'ils tentaient de se sauver, les circonstances précises de leur arrestation en disent long sur les stratégies de survie des déportés. Ce n'est pas seulement vrai pour les tentatives avortées en direction de la France, mais aussi pour les arrestations dans la clandestinité. Les biographies qui figureront dans la seconde partie de cette étude proposeront une description de la manière, du lieu et des circonstances dans lesquelles les femmes, les hommes et les enfants déportés par le X X I e convoi furent arrêtés62. S'y ajouteront des dépouillements statistiques dépassant les histoires individuelles de persécution : d'où venaient les déportés? Combien d'entre eux avaient fui leur pays d'origine, étaient passés dans la clandestinité ou s'étaient procuré de faux papiers? Combien de familles avec enfants, combien d'enfants sans leur proches se trouvaient dans le X X I e convoi ? Quels services allemands, flanqués de leurs acolytes, ont collaboré à l'arrestation ? Certains quartiers ont-ils été méthodiquement fouillés pour y trouver des J uifs ? L es arrestations se concentrent-elles sur certaines périodes? Une synthèse historique établira le bilan des effets réciproques des procédés mis en œuvre par les coupables, qui amenèrent en peu de temps et sans procéder à de grandes rafles des milliers de J uifs de Belgique à Auschwitz, et des stratégies de survie des victimes, dont les tentatives d'échapper se brisèrent finalement au contact de l'inexorable machine à tuer allemande.

62 À paraître dans $L$ es Cahiers de la $M$ émoi re contemporainen ${ }^{\circ} 8$. 


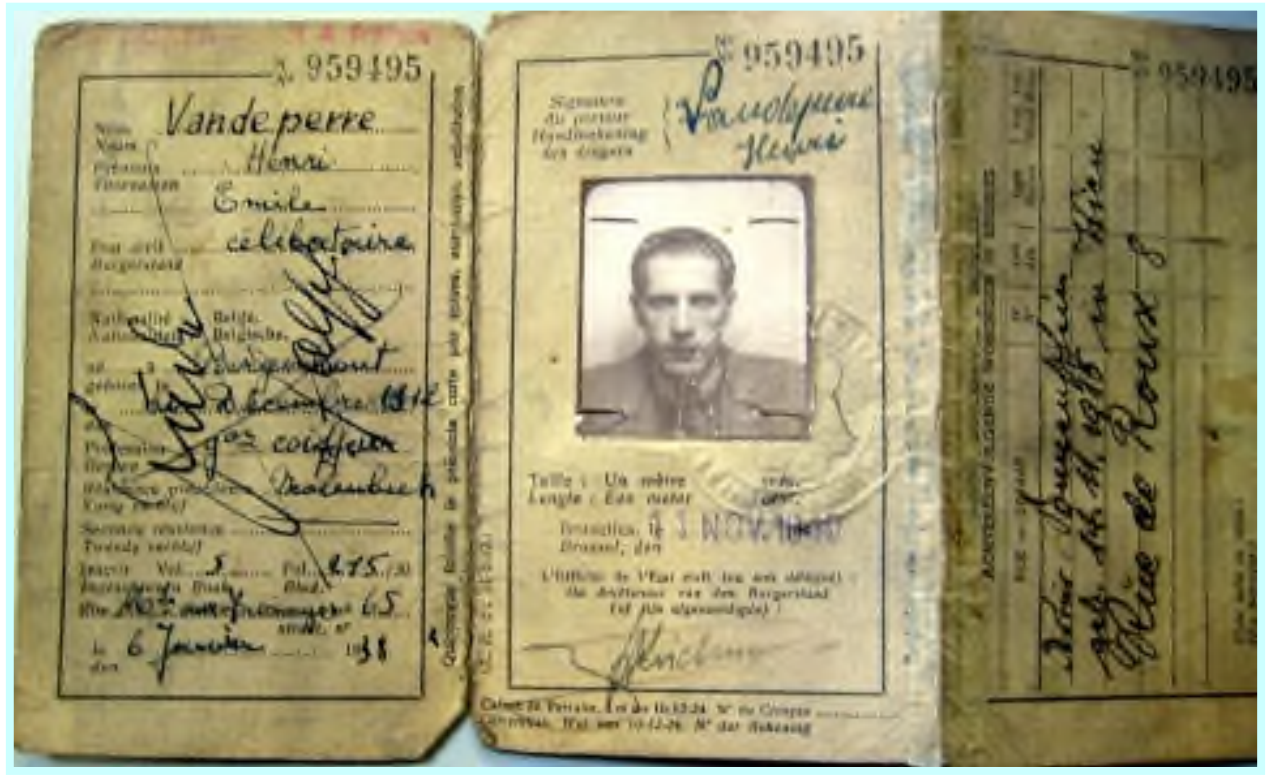

L e faux passeport d'A rthur Sonnenschein.

(c) MJ DR 


\section{HAMBURG-AMERIKA LINIE}

(Compatite Hamburgaesa Americana)

\section{No. 15612}

\section{Klasse f. Clase $t=$ Class}

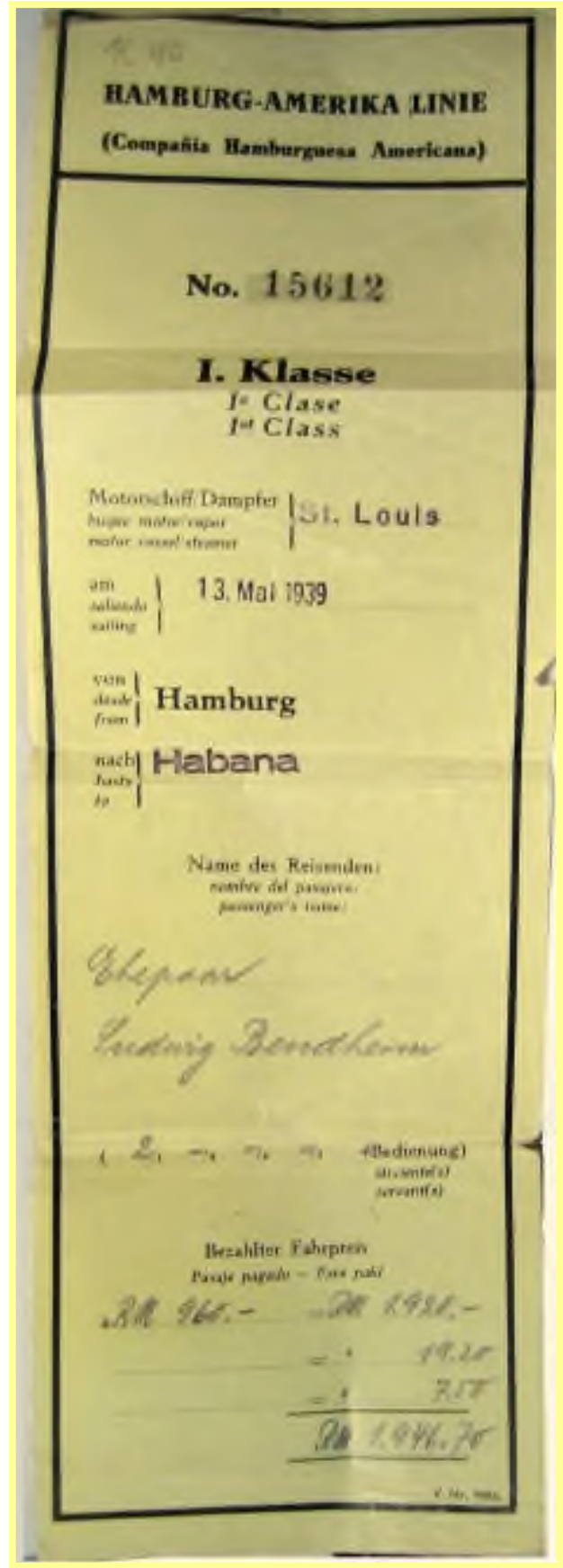

Le billet du couple Bendheim pour une traversée vers Cuba à bord du St. Louis, 13 mai 1939.

$$
\text { (c) MJ DR }
$$



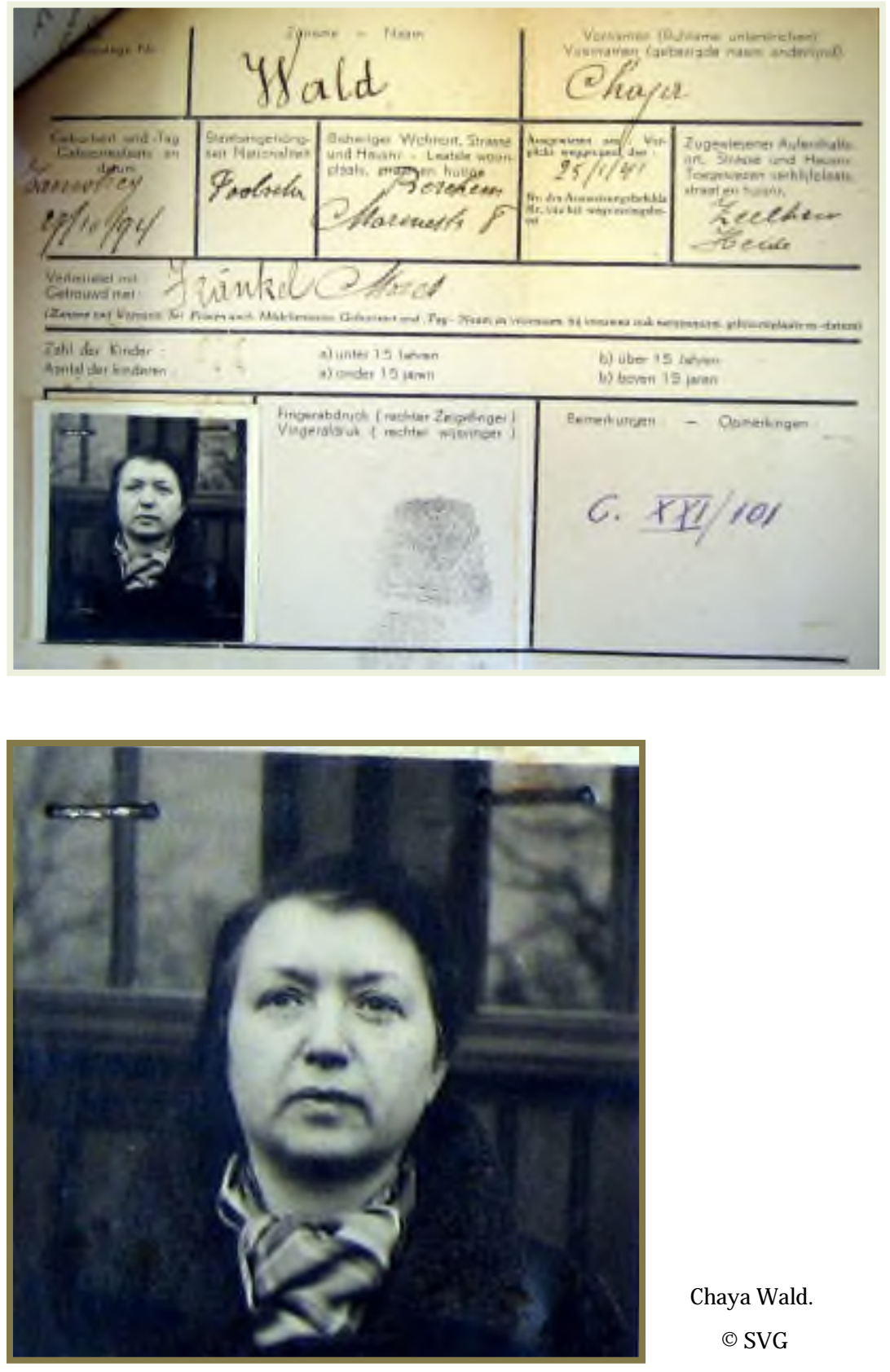

Chaya Wald.

(c) SVG 


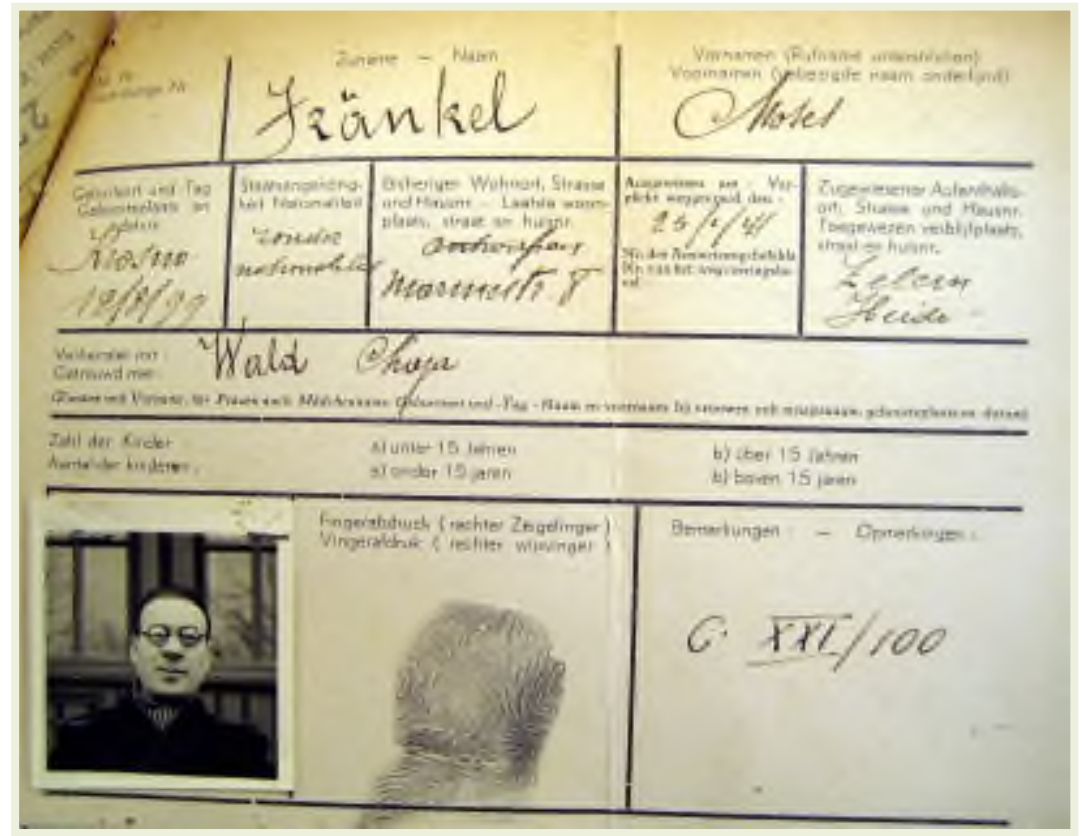

M oses F ränkel.

(c) SVG

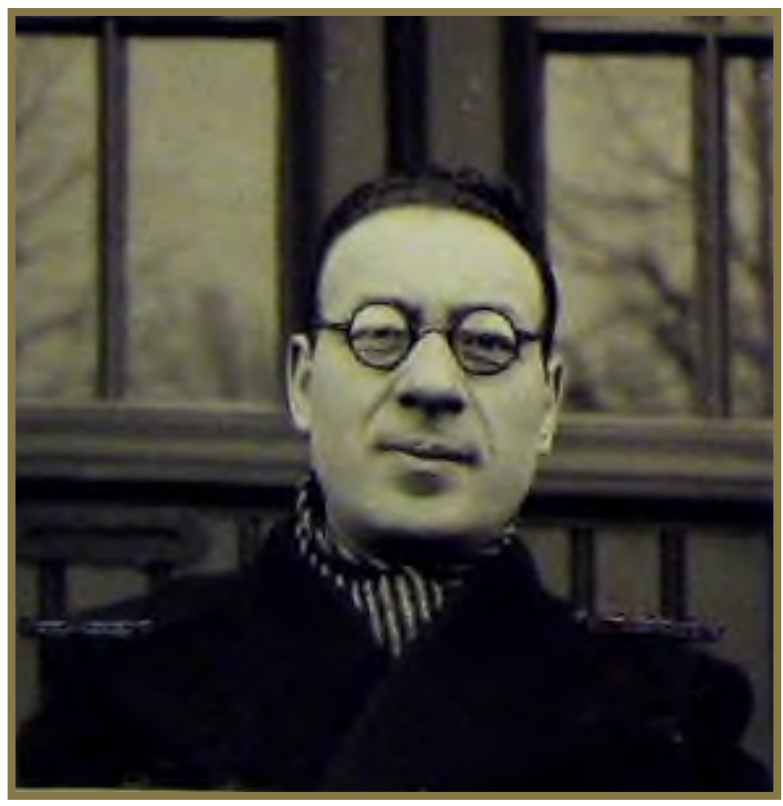



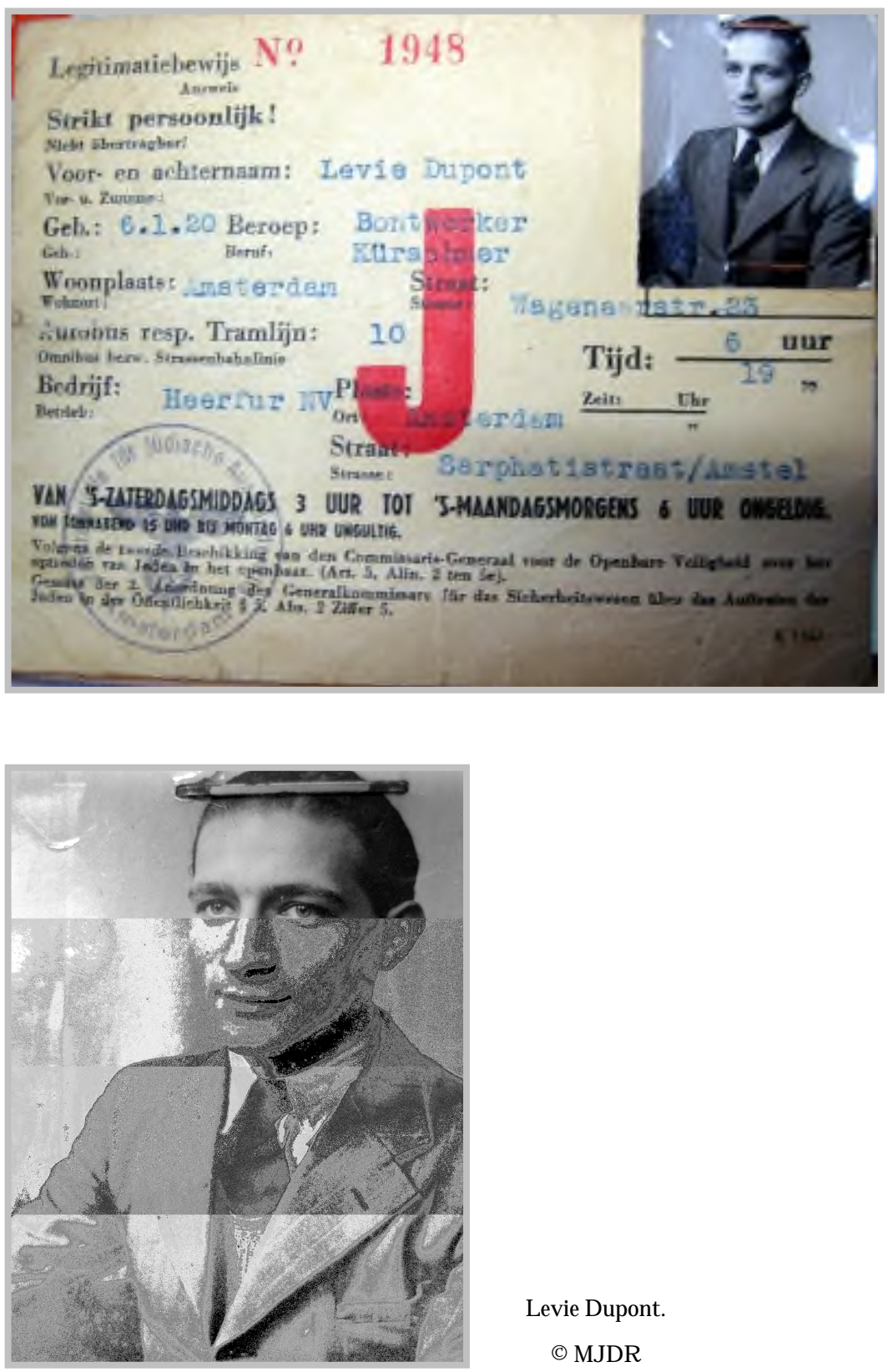

L evie D upont.

(c) MJ DR 


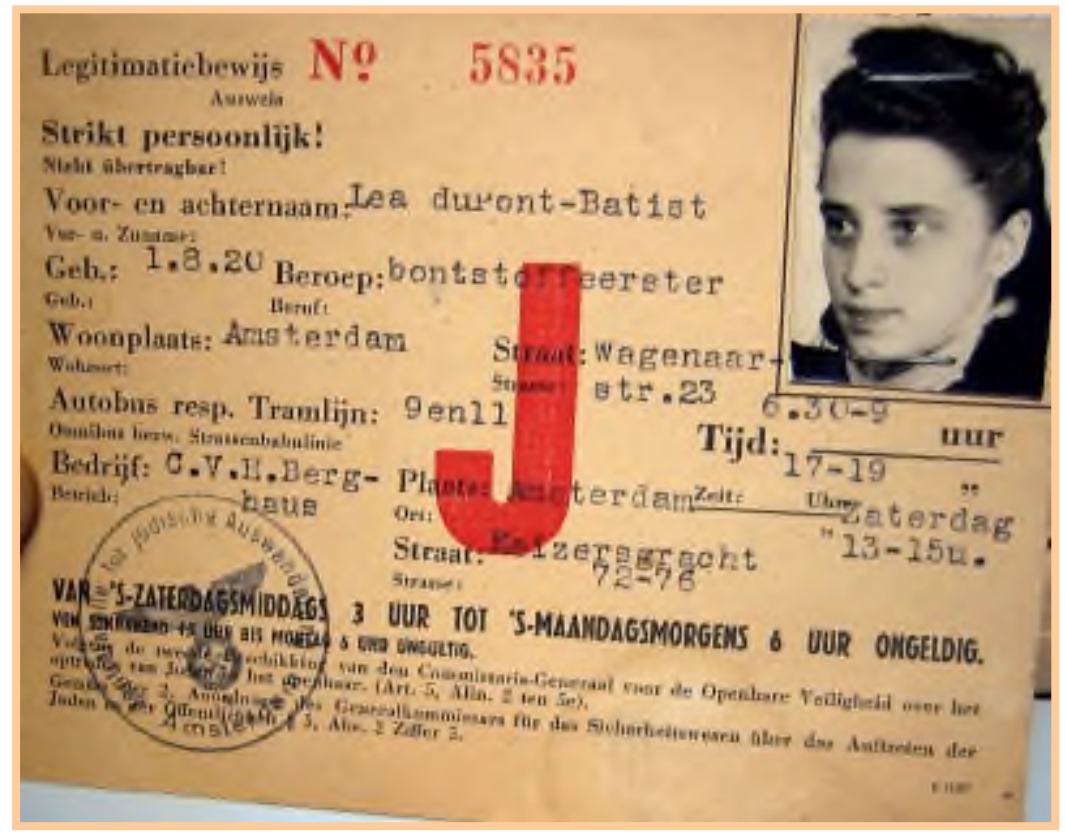

L éa D upont-B atist

(c) MJ DR

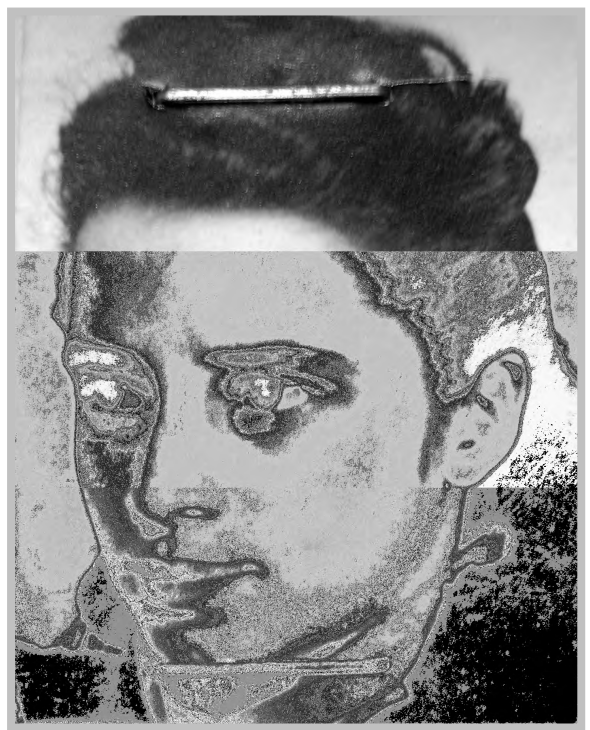



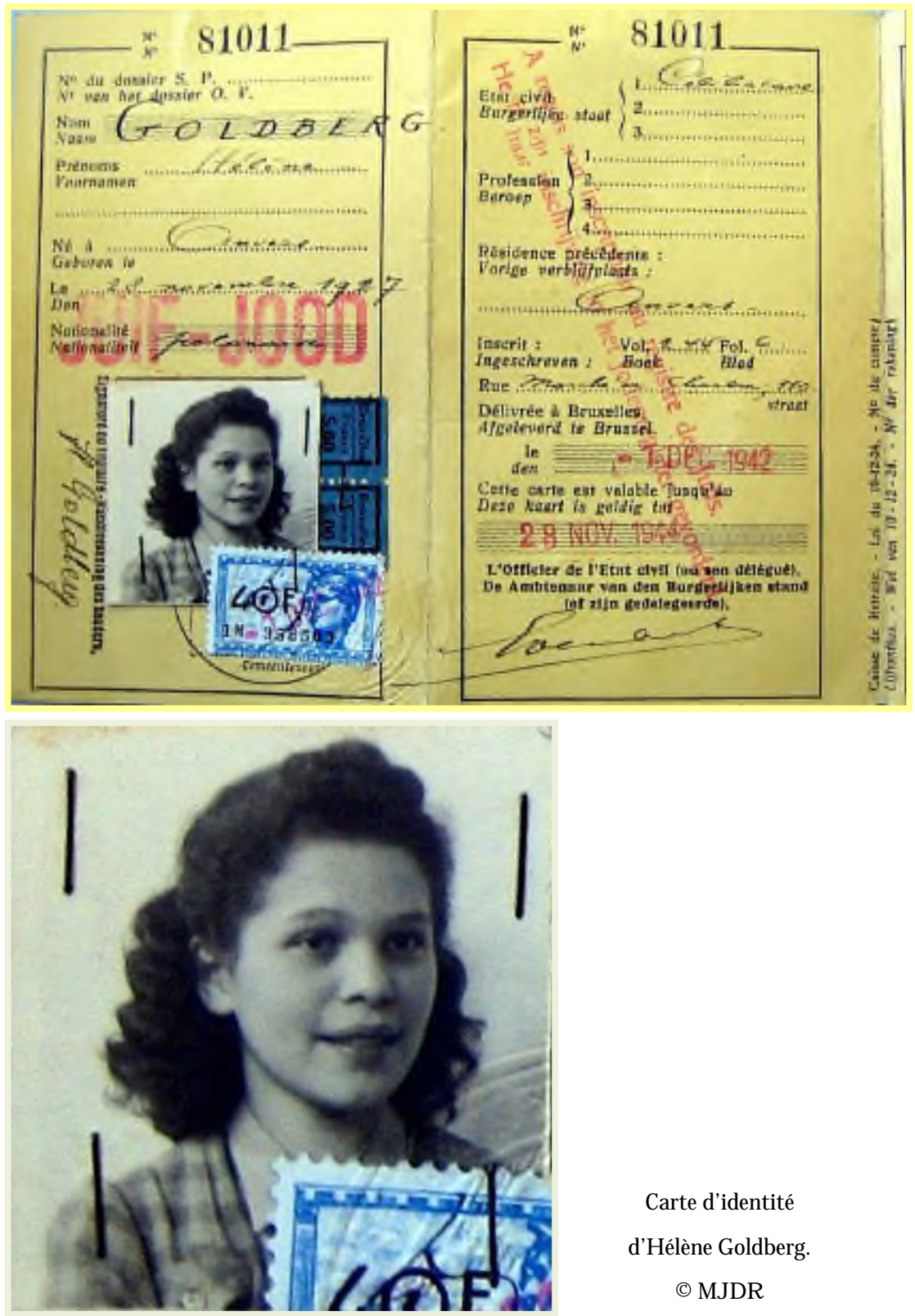

\section{Carte d'identité}

d'H élène Goldberg.

(c) MJ DR 


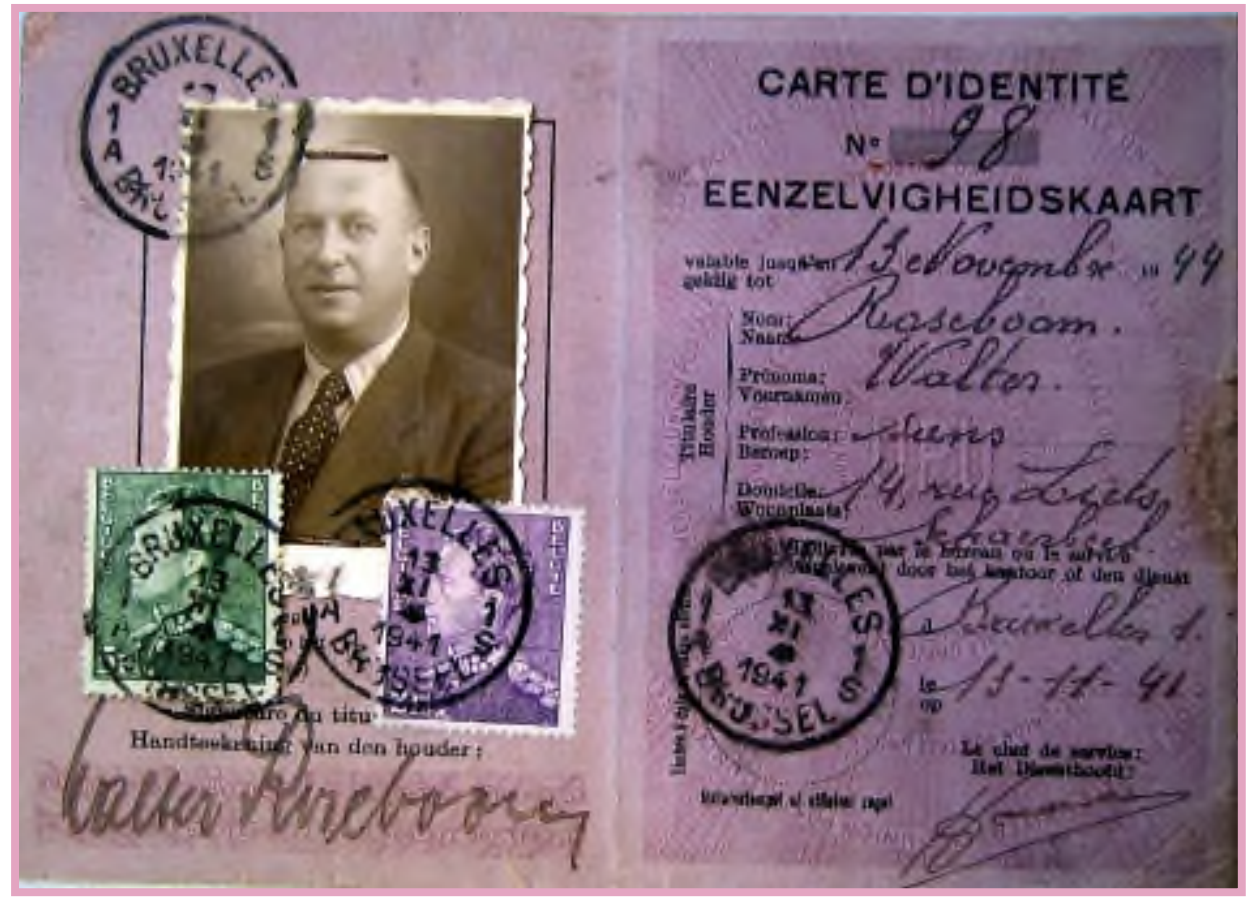

W alter R oseboom.

(c) MJ DR 